GENERAL INSTRUCTION

- Authors: Carefully check the page proofs (and coordinate with all authors); additional changes or updates WILL NOT be accepted after the article is published online/print in its final form. Please check author names and affiliations, funding, as well as the overall article for any errors prior to sending in your author proof corrections.

- Authors: We cannot accept new source files as corrections for your article. If possible, please annotate the PDF proof we have sent you with your corrections and upload it via the Author Gateway. Alternatively, you may send us your corrections in list format. You may also upload revised graphics via the Author Gateway.

QUERY

Q1. Author: Please confirm or add details for any funding or financial support for the research of this article. 


\title{
Time-Domain Terahertz Imaging of Layered Dielectric Structures With Interferometry-Enhanced Sensitivity
}

\author{
Valeri A. Mikhnev ${ }^{\circledR}$, Senior Member, IEEE, Sergey N. Vainshtein, \\ and Juha T. Kostamovaara ${ }^{\circledR}$, Senior Member, IEEE
}

\begin{abstract}
This article presents a time-domain imaging technique for layered dielectric slabs using a solid-state wavelet generator with subterahertz carrier frequency. The technique utilizes the dual nature of a wavelet, i.e., both the applicability of time-offlight measurements and the ability of wavelets to interfere in thin dielectric layers at a carrier frequency that is preserved in spite of the ultrawideband character of the signal. This results in a very high sensitivity of the time delay of the resultant pulse to variations in the effective thickness (thickness $\times$ refractive index) of the dielectric layer. It is shown using a plane-wave analysis of the pulse propagation that under certain conditions, this sensitivity enhancement can reach an order of magnitude. The experimental setup for the reflection-mode operation is described and its performance in the discrimination of healthy and malignant tissues and in the detection of corrosion under paint is demonstrated.
\end{abstract}

Index Terms-Biomedical imaging, interferometry, layered dielectric structures, nondestructive testing, solid-state terahertz (THz) pulsed source, $\mathrm{THz}$ active imaging.

$\mathbf{T}$ HANKS to the transparency of most nonconductive materials in the subterahertz $(\mathrm{THz})$ frequency range and good spatial resolution, $\mathrm{THz}$ waves have found a wide range of applications in imaging and sensing in recent years [1]-[3]. This imaging technology has demonstrated promising results in nondestructive inspection [4], [5], security imaging applications [6], food safety testing [7], etc.

The nonionizing energies of sub- $\mathrm{THz}$ photons also give rise to multiple applications in biomedical imaging [8]-[10]. THz waves are sensitive to water content and thus, its variation within tissue samples can be utilized for medical diagnostics. The contrast in dielectric properties between healthy and malicious tissues [11]-[13] caused by water content variation has been used for the detection of skin, colon, and breast cancer, even

Manuscript received February 14, 2020; revised May 14, 2020; accepted June 5, 2020. This work was supported by the Academy of Finland under Contract 307362 (Center of Excellence in Laser Scanning Research) and Contract 310152. (Corresponding author: Valeri A. Mikhnev.)

The authors are with the Faculty of Information Technology and Electrical Engineering, Oulu University, FIN-90014 Oulu, Finland (e-mail: v.mikhnev@ieee.org; sergey.vaynshteyn@oulu.fi; juha.kostamovaara@oulu.fi).

Color versions of one or more of the figures in this article are available online at https://ieeexplore.ieee.org.

Digital Object Identifier 10.1109/TTHZ.2020.3003500 though the difference in hydration is not the only mechanism responsible for this contrast [14].

Contactless $\mathrm{THz}$ measurement technology has also proven to be highly suitable for industrial applications in the inspection of layered nonmetallic materials, such as plastic, resin, ceramics, and paint coatings. Accurate evaluation of the refractive index and/or thickness of every layer can be performed by using a variety of time-domain and frequency-domain techniques. Relatively thick layers with clear separation of $\mathrm{THz}$ pulses reflected from the layer interfaces can be characterized by simple time-of-flight and phase-slope methods [5], [15], and even better results have been obtained with model-based optimization routines aimed at the best fit between numerical and experimental data [16]-[18]. In very thin stratified structures, however, the received $\mathrm{THz}$ waveforms become difficult to interpret due to overlapping of the partial pulses reflected from the layer interfaces. In this case, extraction of the parameters of interest requires more sophisticated methods based on the solution of an inverse problem or iterative tree algorithm [19].

Most of these advances have been enabled by the discovery of the $\mathrm{THz}$ time-domain spectroscopy method, but in spite of its success, this method has well-known drawbacks, such as the bulky and costly laser-based instrumentation, low output power, and a rather long acquisition time.

Considerable developments have been made in the past decade to both continuous-wave [20]-[23] and pulsed [24]-[33] solid-state components that can be expected to drive the system costs down and promote applications of the THz-time-domain imaging (TDI) technology. Ultrawideband (UWB) millimeterwave and sub-THz pulse generators that are well suited for high-frame-rate imaging are also popular in radar and wireless communications, where a variety of carrier-based and noncarrier-based architectures have been proposed. In the carrier-based scheme, a high-frequency carrier is modulated with a short pulse, producing a high-frequency wavelet, typically with a Gaussian envelope [24]-[25]. A higher power efficiency is offered by noncarrier-based wavelet generators that are built using various schemes such as bandpass shaping of ultrashort pulses [26], edge-combining [27] and pulse-combining [28] methods, and direct digital-to-impulse conversion [29], to name just a few. Gaussian-envelope wavelets can also be generated by 
circuits based on a resonant tunneling diode (RTD) [30]. A timedomain system for material characterization employing this type of pulsed source has already been reported [31]. As these solid-state devices are fabricated by planar microlithographic technology, they can be scaled up to arrays with integrated antennas suitable for multipixel imaging [21], [22].

Another generator of short sub-THz pulses is based on the concept of collapsing-field domains arising in a GaAs avalanche transistor under certain conditions [32], [33]. This produces a train of Gaussian-envelope wavelets with a peak power of a few milliwatts, a center frequency ranging from 130 to $200 \mathrm{GHz}$, depending on the transistor design, and a duration of 40-100 ps. The single-shot jitter of this source is as low as $\sim 1 \mathrm{ps,} \mathrm{which}$ makes it well suited for applications requiring accurate measurements of the time of flight. It was also noticed that there exists a remarkable interference of the wavelets reflected from the interfaces of layered dielectric structures that considerably affects the time of flight of the resultant pulse. The discovery of interferometric enhancement of the sensitivity was found to be promising for TDI applications.

This article will present a comprehensive analysis of the transmission and reflection of a sub-THz pulse with a Gaussian envelope in multilayered dielectric structures, showing that in a properly configured measurement system, the time delay of the received pulse is very sensitive to the effective thickness of the layers in the stratified structure. The variation in the time delay may be an order of magnitude higher than variation of the intrinsic time delay of the electromagnetic wave in the same dielectric material. Combined with the source jitter of $\sim 1$ ps and pulse averaging, the interferometric enhancement technique provides time-of-flight measurements with subpicosecond resolution. Consequently, this measurement approach based on a low-cost all-electronic solid-state sub-THz source with a bandwidth of about $30 \mathrm{GHz}$ [32] may prove competitive in some TDI applications with bulky and costly THz-TDS systems having a bandwidth of a few THz. The performance of the interferometric enhancement technique has been demonstrated in several simulated and experimental examples, including such complicated tasks as the discrimination of healthy and cancerous areas in freshly excised tissue slices. It has also been applied successfully for the detection of corrosion in metal under paint.

The article is organized as follows. Section II addresses the technique of interferometric enhancement, its conditions and limitations. A reflection-mode measurement setup including the pulsed sub-THz source, lenses, and a Schottky detector mounted in a common beam guide is presented in Section III. The experimental results are presented in Section IV, and Section V concludes this article along with proposals for future work.

\section{DESCRIPTION OF THE METHOD}

The proposed TDI technique is based on time-of-flight measurements of a Gaussian-envelope wavelet reflected or transmitted through a multilayered dielectric slab at normal incidence (see Fig. 1).

The corresponding waveforms can be determined as a convolution of the incident signal waveform with the impulse transfer

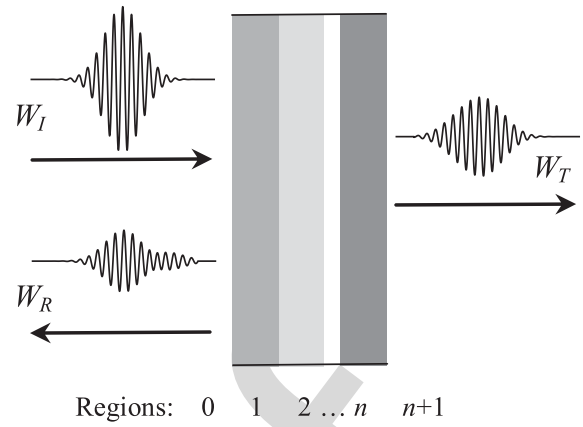

Fig. 1. Geometry of the problem. $W_{I}, W_{R}$, and $W_{T}$ represent incident, reflected, and transmitted wavelets, respectively.

functions of the slab. In the case of a multilayered slab, it is convenient to find its transfer function in the frequency domain with subsequent use of the convolution theorem. Reflected and transmitted wavelets $W_{R}$ and $W_{T}$ can then be calculated using the formulas

$$
\begin{aligned}
& W_{R}(t)=\Phi^{-1}\left\{\Phi\left[W_{I}(t)\right] \cdot R(f)\right\} \\
& W_{T}(t)=\Phi^{-1}\left\{\Phi\left[W_{I}(t)\right] \cdot T(f)\right\}
\end{aligned}
$$

where $W_{I}(t)$ is the incident wavelet, $\Phi$ and $\Phi^{-1}$ are direct and inverse Fourier transforms, and $R(f), T(f)$ are the reflection and transmission coefficients as functions of the frequency $f$.

The functions $R(f)$ and $T(f)$ can both be derived using a concept of scattering transfer matrices that are cascadable and thus, make it easy to deal with multiple layers [34]. Accordingly, a $n$-layer slab is described in the plane-wave case by the product of transfer matrices for the dielectric interfaces and the homogeneous layers between them

$$
\mathbf{A}=\mathbf{I}_{0,1} \mathbf{L}_{1} \mathbf{I}_{1,2} \mathbf{L}_{2} \mathbf{I}_{2,3} \ldots \mathbf{L}_{n} \mathbf{I}_{n,(n+1)}
$$

where $n$ is the number of layers

$$
\begin{aligned}
\mathbf{I}_{(i-1), i} & =\frac{1}{1+r_{(i-1), i}}\left[\begin{array}{cc}
1 & r_{(i-1), i} \\
r_{(i-1), i} & 1
\end{array}\right], i \\
& =1,2, \ldots,(n+1)
\end{aligned}
$$

is the transfer matrix of the interface between regions $(i-1)$ and $i$, and

$$
\mathbf{L}_{i}=\left(\begin{array}{cc}
\exp \left(j \beta_{i}\right) & 0 \\
0 & \exp \left(-j \beta_{i}\right)
\end{array}\right), i=1,2, \ldots, n
$$

is the transfer matrix of the layer $i$. Assuming that the dielectrics of interest are nonmagnetic, the Fresnel coefficients $r_{(i-1), i}$ and the propagation constants $\beta_{i}$ are related to the complex dielectric permittivities of the layers $\varepsilon_{i}$ and their thicknesses $d_{i}$ by

$$
r_{(i-1), i}=\frac{\sqrt{\varepsilon_{\iota-1}}-\sqrt{\varepsilon_{i}}}{\sqrt{\varepsilon_{\iota-1}}+\sqrt{\varepsilon_{i}}} \quad \beta_{i}=\frac{2 \pi f}{c} d_{i} \sqrt{\varepsilon_{i}}
$$

where $c$ is the velocity of light

Once the complete matrix $\mathbf{A}$ of the multilayered structure is calculated, the frequency-domain transfer functions $R(f)$ and $T(f)$ required in (1) can be obtained as

$$
R(f)=A_{21} / A_{11} \quad T(f)=1 / A_{11}
$$




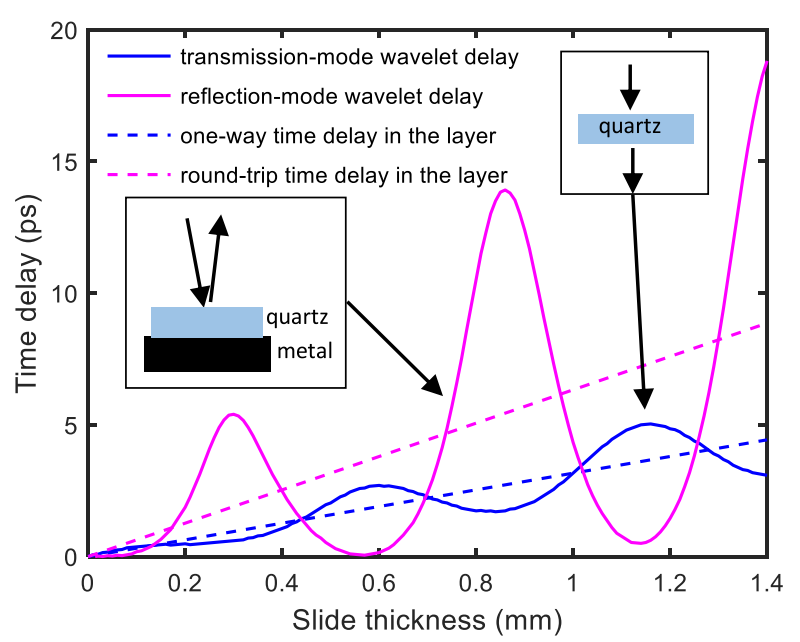

Fig. 2. Calculated time delay of a Gaussian-envelope wavelet (center frequency $135 \mathrm{GHz}$, FWHM 50 ps) transmitted through a quartz layer with a dielectric permittivity $\varepsilon=3.8$ (blue line, geometry shown in the right inset) and reflected from a metal-backed quartz layer (magenta line, geometry is shown in the left inset) versus the layer thickness. The dashed blue and dashed magenta lines show the time delays in the layer as they would be in the absence of interference.

and the final results for the transmitted and reflected wavelets are now accessible from (1).

For transmission-mode modeling, both of the half-spaces (regions 0 and $(n+1)$ in Fig. 1) confining the multilayered slab are assumed to be air. The reflection mode with a metal-backed layer is realized by setting $r_{n,(n+1)}=-1$, implying that the region $(n+1)$ is metal, and then, $W_{T}$ disappears.

In order to obtain the time-delay values comparable with the experimental data, one has to subtract from the propagation time of the wavelet obtained from (1) the propagation time in an air layer of the same thickness as the dielectric sample. This is a consequence of the conventional calibration of the time-delay measurement needed to subtract the time delay in the sensing head, connecting cables, etc. As the initial time delay measured in the experimental setup in the absence of a sample also includes the time delay in the air layer occupied by the dielectric sample at that time, correct comparison requires removing the corresponding time delay from the simulated results, too. In the transmission mode, this means subtraction of a term $\Delta t=\sum d_{i} / c$, while in the reflection mode for a metal-backed layer, the doubled term $2 \Delta t$ needs to be subtracted due to the round trip of the electromagnetic wave in the layer.

The method described above suits well to obtain transmitted and reflected pulses in many practical scenarios with multilayered structures in biomedical imaging and nondestructive testing. Consider first the case of a single layer as far as both its transmission and reflection geometry is concerned. The time delay calculated for the wavelets transmitted through a single dielectric layer and reflected from the same layer placed on a metal substrate is shown in Fig. 2. A Gaussian wavelet with a carrier frequency of $135 \mathrm{GHz}$ and a full width at half-maximum (FWHM) of 50 ps has been adopted here as $W_{I}$.

As seen in Fig. 2, the time delay of both transmitted and reflected wavelets as a function of the layer thickness resembles an interferogram despite the fact that the incident wavelet fulfills the definition of a UWB signal. One must keep in mind, however, that this interferogram is unusual, since the time delay rather than the amplitude is plotted here.

The observed behavior of the time delay can be explained by the interference of the wavelets reflected from the layer interfaces. Thus, in the case of reflection-mode operation with a metal-backed layer, the wavelet reflected from the first interface of the layer overlaps with the leading edge of the wavelet reflected from the metal surface that arrives later.

These wavelets can interfere constructively or destructively, depending on the electrical thickness of the layer. Destructive interference suppresses the leading edge of the resultant pulse that appears as its additional delay, but when the interference is constructive, the leading edge swells up so that the resultant pulse appears to be not delayed at all, e.g., at layer thicknesses of 0.57 and $1.14 \mathrm{~mm}$ in Fig. 2. Incidentally, the specified thicknesses correspond exactly to the half-wavelength and one-wavelength quartz layers at a frequency of $135 \mathrm{GHz}$, respectively. This is clear evidence of the interference nature of the graph in Fig. 2. Alternatively, the maxima of the time delay in the reflection mode may appear when the effective thickness of the layer is equal to an odd number of quarter-wavelengths.

The oscillations in the time delay of the Gaussian wavelet versus the electrical thickness of the dielectric layer can be used for the imaging of electrically thin dielectric structures. To this end, the operating point should be chosen on one of the slopes of the "interferogram" in Fig. 2. Then, small variations in either the refractive index or the thickness of the layer will result in pronounced variations in the time delay.

As the oscillations in the time delay are much stronger in the reflection mode, this mode is preferred in further considerations because of the higher expected sensitivity. Nevertheless, the transmission mode can be useful, too. For instance, a high spatial resolution can be achieved in the transmission mode by using a focused beam and a detector with a small aperture. There is also some gain in sensitivity in this mode, although lower than in the reflection mode.

The time delay in the resultant pulse has been determined in the above calculations from the position of its maximum. Detection of the position of the rising edge of the pulse is preferable in the experimental studies for the sake of better measurement accuracy, but in this case, the Gaussian shape of the received wavelet should be preserved. The wavelet envelopes were calculated for a set of maxima [see Fig. 3(a)] and minima [see Fig. 3(b)] in the reflection mode using the geometry of the left inset in Fig. 2 and the same characteristics of the probing wavelet. For clarity, only two maxima and two minima of the time delay are shown in Fig. 2, whereas in Fig. 3(a), the envelopes of the first four maxima are plotted (the corresponding quartz thicknesses are $0.28,0.85,1.42$, and $1.99 \mathrm{~mm}$ ).

The time scale in Fig. 3 is selected so that the apex of the probing wavelet corresponds to zero time. It is seen in Fig. 3(a) that the wavelets of the first two maxima preserve their Gaussian shape well. At the next maxima, the pulse shape is distorted and widened, although it suits quite well for time-of-flight measurements. 


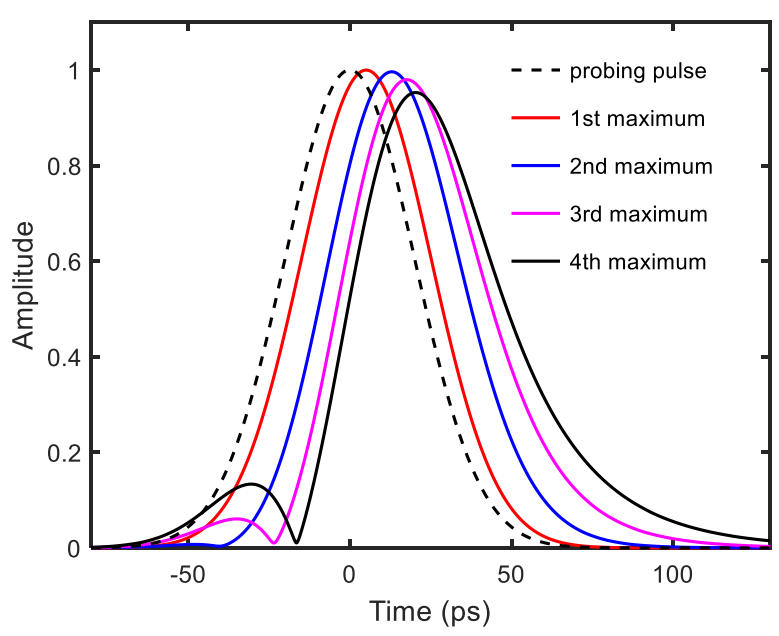

(a)

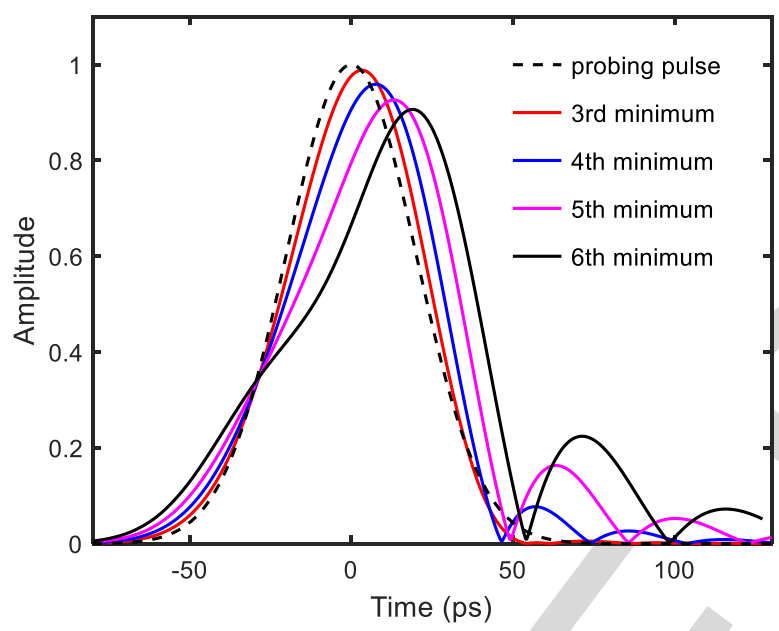

(b)

Fig. 3. Shapes of the envelopes of received wavelets in the reflection mode of operation using the geometry shown in the left inset in Fig. 2 and the same characteristics of the probing wavelet. (a) Shapes corresponding to a set of the first four maxima of the time delay (thicknesses of quartz substrate are 0.28 , $0.85,1.42$, and $1.99 \mathrm{~mm}$ ). (b) Shapes corresponding to a set of minima of the time delay numbered 3-6 (corresponding quartz thicknesses are 1.71, 2.28, 2.85, and $3.42 \mathrm{~mm}$ ).
In Fig. 3(b), the shapes of the received pulses for the minima numbered 3-6 (with corresponding quartz thicknesses of 1.71, $2.28,2.85$, and $3.42 \mathrm{~mm}$ ) are plotted. The wavelet shapes for the first and second minima (layer thicknesses 0.57 and $1.14 \mathrm{~mm}$ ) are not shown here as they are graphically indistinguishable from the probing wavelet. As in Fig. 3(a), the pulses of the first minima in Fig. 3(b) keep their Gaussian shapes, being almost exact replicas of the probing wavelet. At greater thickness of the substrate, however, the shape of the received pulse is rapidly distorted and the slope of the time-delay interferogram does not increase any longer. Hence, for implementation of the method, the dielectric substrate must be thick enough to achieve better sensitivity, while at the same time avoiding any marked deviation of the received pulse from the Gaussian shape.

It is intuitively expected from Fig. 2 that the areas of high sensitivity to a thin dielectric layer placed over the metal-backed

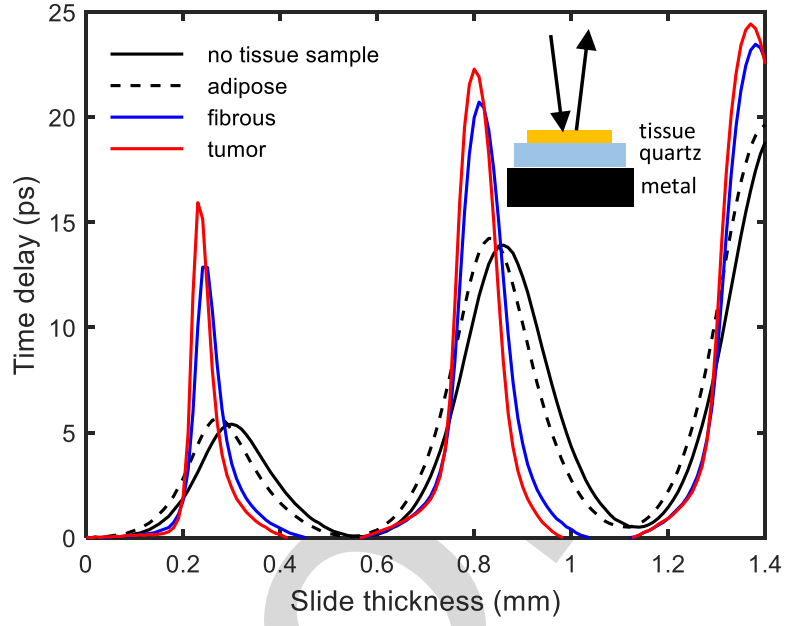

Fig. 4. Time delay of the Gaussian-envelope wavelet (center frequency $135 \mathrm{GHz}, \mathrm{FWHM} 50 \mathrm{ps}$ ) versus thickness of the quartz slide calculated for the "tissue-quartz-metal" structure in the reflection mode. The tissue slice thickness is $50 \mu \mathrm{m}$, and the dielectric permittivities of the three tissue types (adipose, fibrous, and tumorous) are taken to be $\varepsilon_{a}=2.4-\mathrm{j} 1.0 ; \varepsilon_{f}=3.6-\mathrm{j} 5.3$; $\varepsilon_{t}=4.4-\mathrm{j} 6.2$, respectively. Inset: Simulation model.

slide will correspond to both slopes in the time-delay curve. To verify this, we calculated the time-delay variations for three types of fresh tissue slices-adipose, fibrous, and tumorous (see Fig. 4), the dielectric permittivity data for which are taken from [35]. The simulation model is shown in the inset in Fig. 4.

It is seen from the simulation results that the sensitivity of the method to the dielectric properties of a thin layer does indeed increase considerably, so that the additional round-trip propagation time of the electromagnetic wave in the 50- $\mu$ m layer with a refractive index of the fibrous tissue of $n_{f}=\operatorname{Re} \sqrt{\varepsilon_{f}} \cong 2.25$ can be estimated to be $2 d\left(n_{f}-1\right) \cong 0.4 \mathrm{ps}$, while at some points in Fig. 4, the corresponding time-delay difference reaches 8-9 ps. This constitutes the principle of interferometric enhancement for pulsed time-domain imaging. One must keep in mind that the results obtained here correspond to the parameters of pure tissues whereas in real slices, the tissue types may be intermingled.

The slice thickness should be chosen as a tradeoff between sensitivity and the interference conditions. Too thin a tissue sample will make a low contribution to the reflected signal, while too thick a sample will suppress the effect of the interference. For the tissues considered here and the signal parameters specified in Fig. 4, the optimal range of slice thickness should be about 50 to $100 \mu \mathrm{m}$.

The most complicated case in breast cancer imaging is the differentiation between fibrous and cancerous tissues, as their dielectric properties are very similar [35]. The estimated round-trip propagation times in $50 \mu \mathrm{m}$ slices of those tissues yield a difference of only $\sim 0.1 \mathrm{ps}$, which is very challenging even for femtosecond laser-based pulsed $\mathrm{THz}$ imaging, but interferometry-enhanced TDI can increase the difference to 1-2 ps (see Fig. 4) if the thickness of the quartz slide is selected properly.

Finally, we should consider the influence of the wavelet center frequency and the dielectric permittivity of the slide on the efficiency of interferometric enhancement (see Fig. 5). 


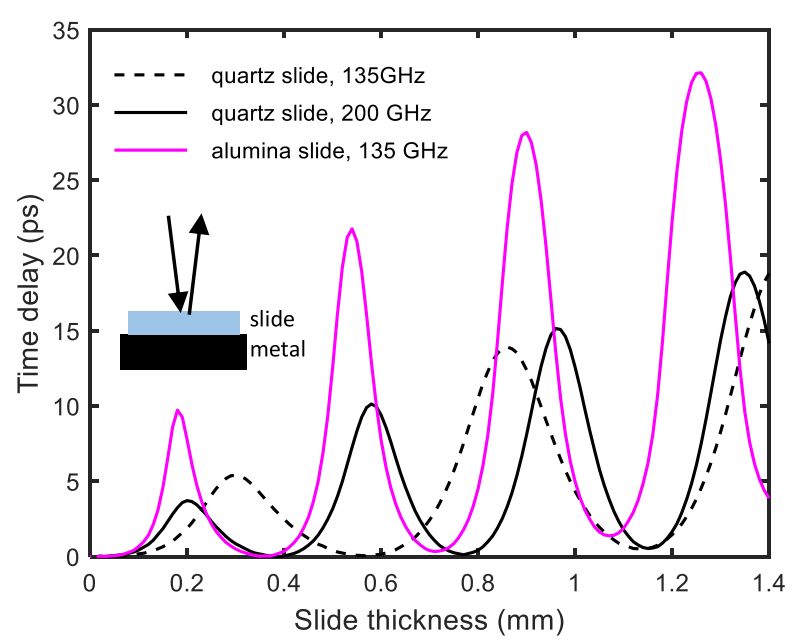

Fig. 5. Calculated time delay of the Gaussian-envelope wavelets versus slide thickness in the reflection mode (see inset) for different slides and center frequency of the wavelet: Quartz slide and frequency of $135 \mathrm{GHz}$ (dashed black); alumina slide and frequency of $135 \mathrm{GHz}$ (magenta); quartz slide and frequency of $200 \mathrm{GHz}$ (solid black).

As the center frequency of the wavelet increases (e.g., by using a 200-GHz pulsed source [33]), more oscillations of the time delay occur in the same interval of thickness that is natural for the interferometry. Consequently, the slopes of the "interferogram" become steeper, leading to a higher sensitivity. A similar effect can also be achieved by replacing quartz with a dielectric having a larger refractive index, e.g., alumina, with dielectric permittivity $\varepsilon=9.6$ (see Fig. 5, magenta line). The use of a high-permittivity substrate is not reasonable in this arrangement, however, despite the attractive steepness of the interference curve. The problem is that the amplitudes of the interfering pulses are comparable in this case, and this may lead to disintegration of the resultant wavelet and thus, to jumps in the measured time delay. Consequently, both the type and thickness of the slide and the frequency of operation must be optimized for any practical scenario by means of preliminary simulations, as in Fig. 4.

The operating point on the inclined section of the time-delay interferogram can also be selected experimentally. To this end, thin quartz slides with a thickness of $0.2-0.3 \mathrm{~mm}$ can be added to the main quartz slide and thus, the substrate thickness corresponding to the highest sensitivity in the inclined section of the time-delay interferogram can be estimated. In addition, it should be kept in mind that there exists an intrinsic limitation to the range of the time delay, which cannot exceed approximately half-FWHM, because the interfering wavelets must overlap well in order to create the required effect.

\section{EXPERIMENTAL SETUP}

The pulsed sub-THz source with an on-chip semicircular bowtie antenna [32] was fabricated on a $100-\mu \mathrm{m}$ GaAs substrate using state-of-the-art heterojunction bipolar transistor (HBT) technology with some modifications. The fabricated chip was mounted on the flat side of a hyperhemispherical high-resistivity

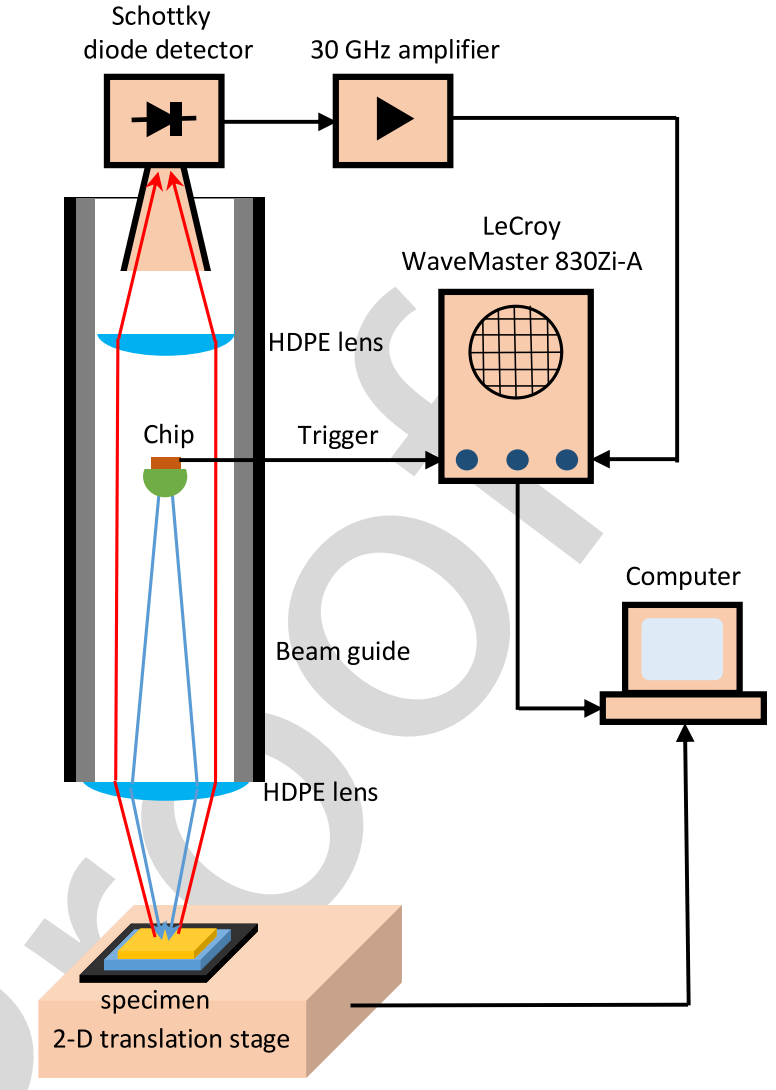

Fig. 6. Reflection-mode experimental setup. Propagation of the incident wave is shown schematically by blue lines, and that of the reflected wave is shown in red. The specimen under test (yellow) is placed on a metal-backed quartz slide (blue).

silicon lens with a diameter of $4 \mathrm{~mm}$ for efficient coupling of the radiation to the free space. The output power of the emitter was measured using a special setup consisting of a large elliptical mirror, a Golay cell, and a set of metal-mesh quasi-optical bandpass filters. The typical peak output power was $\sim 1 \mathrm{~mW}$. The emitter was then placed in the center of a hollow dielectric beam guide supporting a Gaussian beam at frequencies above $100 \mathrm{GHz}$ with low attenuation [36]. A simplified functional scheme of the experimental setup is shown in Fig. 6 .

The pulse is emitted by the chip through the silicon lens and propagates along the beam guide with a diameter of $20 \mathrm{~mm}$. It is then focused by a high-density polyethylene (HDPE) lens (diameter $25.4 \mathrm{~mm}$, focal length $25 \mathrm{~mm}$ ) onto a layered structure including a metal substrate, a quartz slide, and a specimen of the material under test. The reflected wavelet propagates through the HDPE lens in the opposite direction and after some shaping by the beam guide, circumvents the emitter. In the end, it is collected by a waveguide horn antenna with attached zero-bias Schottky detector (Virginia Diodes, 110-170 GHz) matched well at the wavelet center frequency of about $135 \mathrm{GHz}$. To enhance the amplitude of the received signal, another HDPE lens is placed in front of the waveguide horn at a distance that ensures maximum signal transfer from the beamguide mode to the detector.

The detected wavelet envelopes were recorded using a LeCroy WaveMaster 830Zi-A oscilloscope. In the future, we plan to 
replace the oscilloscope with an ultrafast time-to-digital converter, but this is a difficult problem as the oscilloscope is triggered from a current pulse formed in the source supply circuit during avalanche switching simultaneously with the sub- $\mathrm{THz}$ emission, and thus, the single-shot jitter is $\sim 1$ ps. This nevertheless promises high-precision time-of-flight measurements, especially with the use of averaging, although it also imposes strict requirements on the time-to-digital converter. The specimen is mounted on a computer-controlled motorized translation stage and scanned two-dimensionally during data acquisition to form the image.

The spatial resolution was evaluated by a knife-edge method and is about $5 \mathrm{~mm}$, which agrees well with the diffraction limit at the frequency of operation.

The reflection-mode setup design presented here differs from the conventional schemes that employ either a beam splitter or a scheme under oblique incidence [9], [12], [14]. Although there are some losses in our system related to high divergence of the incident beam and partial shielding of the reflected wave by the emitter lens, the reflected signal was still found to be larger than in the usual design based on a beam splitter. Besides, the design shown in Fig. 6 is very compact and robust, as all the critical components are placed in a common metal tube of diameter $40 \mathrm{~mm}$ embracing the hollow dielectric beam guide. This makes it attractive for future practical applications.

\section{EXPERIMENTAL RESULTS}

For experimental verification of the effect of interferometric enhancement in the reflection mode, we prepared a sample of polyethylene terephtalate (PET) with a steplike thickness profile mounted on a metal substrate. PET was chosen due to the relative simplicity of preparing multiple layers of different thicknesses. In our specimen, they varied in thickness between 0 and $1.2 \mathrm{~mm}$ in steps of $0.2 \mathrm{~mm}$. The width of each layer was about 7-8 mm, which is a little larger than the spatial resolution of the experimental setup. Strips of clean metal were left at both ends of the fabricated specimen for performing calibration of the time delay. The time-delay measurements were also performed for several available quartz slides with thicknesses in the range $0.8-1.3 \mathrm{~mm}$, and their results are plotted in Fig. 7 along with the curves obtained by numerical simulations for the center frequency of $135 \mathrm{GHz}, \mathrm{FWHM} 40 \mathrm{ps}$, and the dielectric permittivity of PET $\varepsilon=2.56$ and quartz $\varepsilon=3.8$.

The oscillating behavior of the time delay of the reflected pulse depending on the thickness of the metal-backed layer was demonstrated experimentally for two types of dielectrics. The experimental points agree reasonably well with the results of the numerical simulations, the differences being in most cases about 1-1.5 ps, which is close to the values for the jitter and drift of the pulsed source and digital oscilloscope employed in the experimental setup.

The high sensitivity and picosecond resolution of the time-offlight measurements look promising with respect to resolving the challenging problems of biomedical diagnostics such as differentiation between healthy and malignant tissues. Due to the high absorption of the sub-THz signal in biological materials,

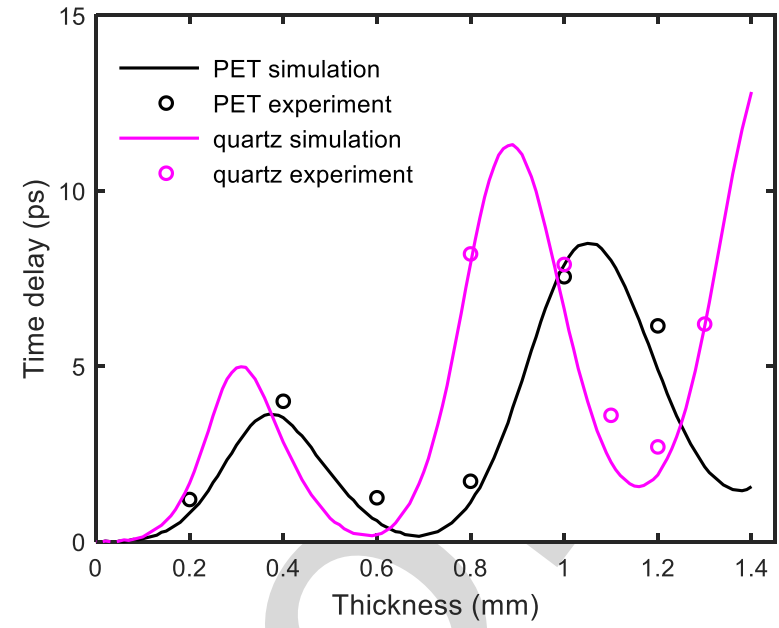

Fig. 7. Time delay of the Gaussian-envelope wavelets (center frequency $135 \mathrm{GHz}, \mathrm{FWHM} 40 \mathrm{ps}$ ) reflected from PET and quartz layers of different thickness. The experimental results are shown by circles. The results of the numerical simulations are also given for comparison (solid lines).

sample preparation must rely either on very thin tissue, with a loss of sensitivity, or on dehydration of the sample through formalin fixing and embedding in paraffin in order to maintain a reasonable transmitted signal. If the pathological analysis of a paraffin-embedded sample shows positive margins of the cancerous area, a second operation will be required, which is highly undesirable for many reasons, whereas if the decision could be made intraoperatively by analyzing freshly excised tissue slices, then the malignant tumor could be removed in a single operation. The results of our first experiments with the breast cancer tissue samples are presented in Fig. 8.

First, the tissue slices provided by Oulu University Hospital were measured in transmission mode, i.e., a 300- $\mu$ m thick slice was mounted on a quartz slide of thickness $1.2 \mathrm{~mm}$. The contrast of the obtained images was very poor for both amplitude and time-of-flight data and thus, no clear correlation with the histology could be found.

The images were then built up using the reflection-mode setup shown in Fig. 6 and much thinner slices, of thickness only $100 \mu \mathrm{m}$. The contrast in time delay measured over the tissue slice increased to $5 \mathrm{ps}$ when the $1.2-\mathrm{mm}$ quartz slide was used [see Fig. 8(b)]. This corresponded to the ascending branch of the "interferogram" in Fig. 7. In another experiment, the operating point was selected on the descending branch of the curve in Fig. 7 by using a quartz slide of thickness $1.0 \mathrm{~mm}$. As a result, the contrast with the time delay recorded over the same tissue slice increased to 13 ps [see Fig. 8(c)].

The area of a pronounced deviation of the time delay from the background values in Fig. 8(b) and (c) corresponds satisfactorily to the histological position of tumorous area [see Fig. 8(a)], although the spatial resolution needs to be improved. We expect that the next designs employing 200-300 GHz sources [33] will solve this problem.

Another promising application for the interferometric enhancement technique is the detection of corrosion under paint. Identifying corrosion before it becomes visible can help to 


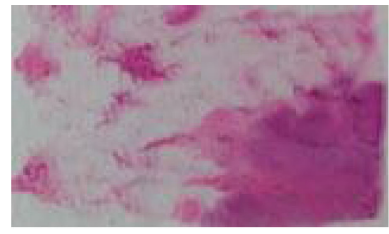

(a)

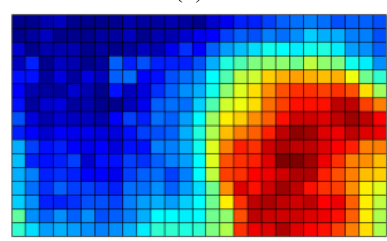

Time delay, ps

(b)

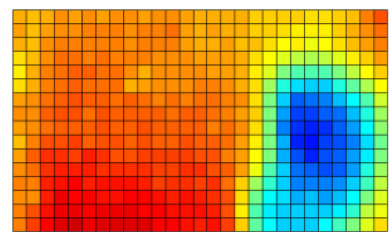

Time delay, ps

(c)

Fig. 8. Images of successive slices of the same fresh-frozen breast tumor tissue. (a) Histology, where adipose tissue is transparent, fibrous tissue is pink and intermingled with adipose, and tumor tissue is violet-pink. (b) Time-of-flight reflection-mode image obtained with a $100-\mu \mathrm{m}$ slice placed on a $1.2-\mathrm{mm}$ quartz slide. (c) Time-of-flight reflection-mode image obtained with the $100-\mu \mathrm{m}$ slice placed on a 1.0-mm quartz slide. The time-delay values in (b) and (c) mean a delay difference related to a selected operating point. The size of the grid cell in (b) and (c) is of $1 \mathrm{~mm} \times 1 \mathrm{~mm}$.

avoid potential structural problems and minimize repair costs. Corrosion causes roughness of a metal surface that in turn results in marked scattering of electromagnetic waves of short wavelengths. Consequently, corroded areas can be detected as areas of lower reflectivity in the sub-THz reflection image obtained at normal incidence [37], [38].

The applicability of the interferometric enhancement technique to the detection of hidden corrosion has been examined using the experimental setup shown in Fig. 6 and a specially processed specimen of homogeneously rusted steel in which the rust had been partially removed to form alternating strips of rusty and clean metal approximately $15 \mathrm{~mm}$ in width [see Fig. 9(a)].

The specimen was placed on the translation stage as shown in Fig. 6 and scanned twice in the same direction, across the alternating strips, before and after painting. Both the amplitude and time delay of the reflected Gaussian-envelope pulse with a center frequency of $135 \mathrm{GHz}$ were recorded. The results are plotted in Fig. 9(b) and (c).

For easy comparison, a common scale is used on the $x$-axis throughout Fig. 9. The specimen was then painted using a conventional off-the-shelf fast drying acrylic paint, and the thickness of the coating, measured as the difference in the total thickness of the specimen before and after painting, was found to be about $200 \mu \mathrm{m}$. As shown clearly in Fig. 9(b), corrosion reduces the reflectivity of a metal, a fact that can be used for the detection of corroded areas using two-dimensional (2-D) mapping, as is done in [37] and [38]. The rust under the paint can

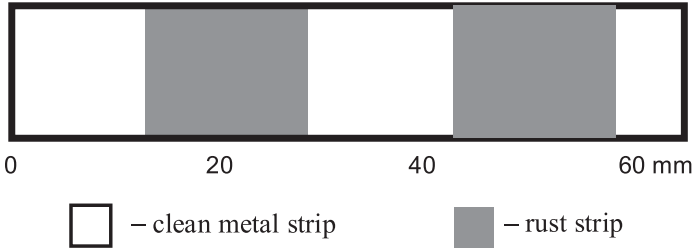

(a)

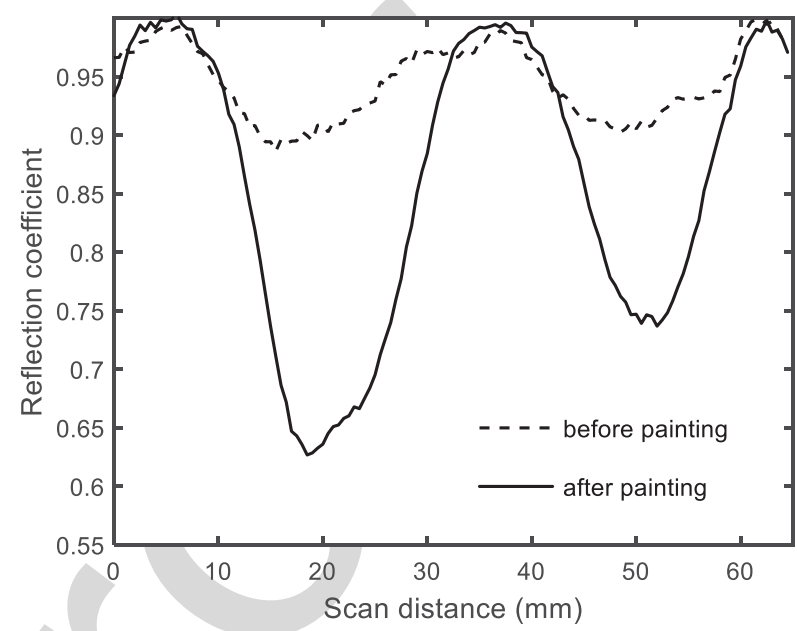

(b)

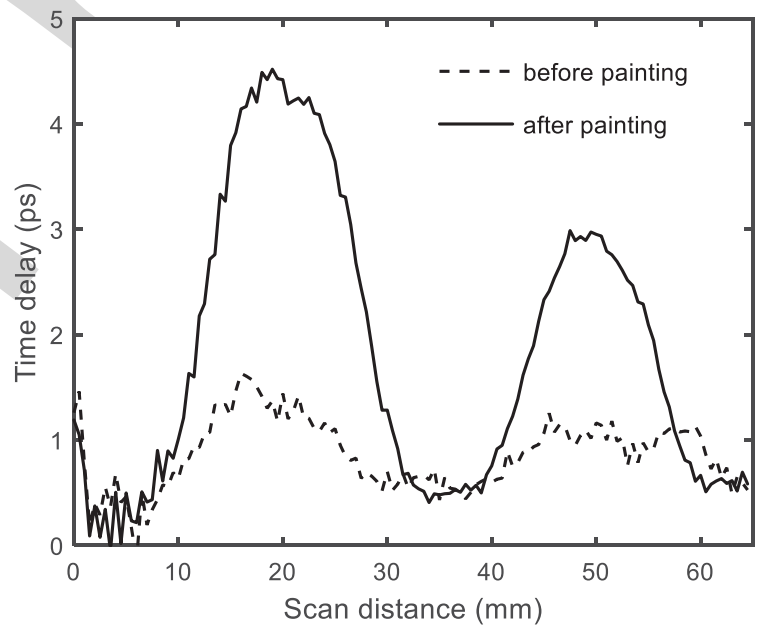

(c)

Fig. 9. Inspection of corrosion under paint by the interferometric enhancement technique. (a) Scheme of the specimen under test with rusty and clean metal areas. (b) Amplitude of the reflected Gaussian-envelope pulse versus scan distance. (c) Time delay of the same pulse versus scan distance.

be detected even more easily due to the stronger scattering of the incident wave on the rough surface caused by the rust. Along with this effect, one can observe that the Gaussian-envelope pulse reflected from the rusted area has been delayed by $3-5 \mathrm{ps}$ [see Fig. 9(c)]. This delay correlates well with the presence of the rust under the paint and considerably exceeds the time delay that could be expected from a round-trip propagation of the electromagnetic wave in the paint layer. Thus, the additional round-trip time delay in a paint layer with a refraction index $n_{p} \approx 1.6$ [39] and a thickness $d=0.2 \mathrm{~mm}$ can be estimated to be $2 d\left(n_{p}-1\right) \cong 0.8$ ps which is $4-6$ times smaller than the time delay measured in the experiment. 
This anomalous behavior of the time delay can be explained by the interferometric enhancement technique. It can be deduced from the previous analysis that the sweep range of the time of flight of the reflected pulse depends on the ratio of the coefficients of reflection from the dielectric layer interfaces. The closer the amplitudes of the interfering wavelets are, the larger will be the range of the time-delay sweep (naturally within the above-mentioned limits $\pm 1 / 2$ FWHM). In particular, it is for this reason that the sweep of the curve for the alumina slide in Fig. 5 is much larger than that for the quartz slide.

In the case of rust under paint, the decrease in the coefficient of reflection from the paint-metal interface caused by the rust also drives the amplitude of the corresponding wavelet closer to that of the wavelet reflected from the air-paint interface. Accordingly, the time delay of the resultant wavelet in Fig. 9(c) is enhanced by interferometry and this enhancement correlates with the drop in reflection coefficient in Fig. 9(b). Thus simultaneous measurement of both the amplitude and time delay of the sub-THz pulse with Gaussian envelope provides more information about the rusted area and shows still greater promise as a reliable tool for the detection of corrosion under paint.

\section{CONCLUSION}

The technique of interferometry-enhanced TDI presented here is intended for millimeter-wave and THz imaging systems based on Gaussian-envelope wavelet sources. One source of this kind is used here, but the technique can also be implemented with wavelet generators employing other principles such as edge- and pulse-combining methods, RTD generators, and others that are being rapidly developed nowadays. Compact and affordable TDI systems using solid-state sources and detectors promise to move $\mathrm{THz}$ imaging from the research lab to real-world applications, e.g., to clinical intraoperational diagnostics. The technique can also be applied to some nondestructive inspection problems as demonstrated in the example of the detection of corroded areas under paint.

\section{ACKNOWLEDGMENT}

The authors would like to thank Dr. J. Näpänkangas and Dr. R.Blanco-Sequeiros of Oulu University Hospital for providing ex vivo tissue samples for the $\mathrm{THz}$ measurements. Permission for the use of the breast cancer tissue samples (reg. no. 59-2009) was obtained from the Ethical Board of Oulu University Hospital, Finland.

\section{REFERENCES}

[1] D. M. Mittleman, "Twenty years of terahertz imaging [Invited]," Opt. Express, vol. 26, pp. 9417-9431, Apr. 2018, doi: 10.1364/OE.26.009417.

[2] D. Saeedkia, Handbook of Terahertz Technology for Imaging, Sensing and Communications. Cambridge, U.K.: Woodhead, 2013.

[3] D. Suzuki, S. Oda, and Y. Kawano, "A flexible and wearable terahertz scanner," Nature Photon., vol. 10, pp. 809-813, Dec. 2016, doi: 10.1038/nphoton.2016.209.

[4] I. Amenabar, F. Lopez, and A. Mendikute, "In introductory review to THz non-destructive testing of composite mater," J. Infrared Millim. THz Waves, vol. 34, pp. 152-169, Feb. 2013, doi: 10.1007/s10762-012-9949-z.
[5] F. Ellrich et al., "Terahertz quality inspection for automotive and aviation industries," J. Infrared Millim. THz. Waves, vol. 41, pp. 470-489, 2020, doi: 10.1007/s10762-019-00639-4.

[6] K. Cooper et al., "THz imaging radar for standoff personnel screening," IEEE Trans. THz Sci. Technol., vol. 1, no. 1, pp. 169-182, Sep. 2011.

[7] W.-H. Lee and W. Lee, "Food inspection system using terahertz imaging," Microw. Opt. Techn. Lett., vol. 56, pp. 1211-1214, May 2014, doi: $10.1002 / \mathrm{mop} .28303$.

[8] Y. Sun et al., "A promising diagnostic method: Terahertz pulsed imaging and spectroscopy," World J. Radiol., vol. 3, pp. 55-65, Mar. 2011, doi: $10.4329 / 2$ Fwjr.v3.i3.55.

[9] E. Pickwell-MacPherson and V. P. Wallace, "Terahertz pulsed imaging-A potential medical imaging modality?" Photodiagnosis Photodyn. Therapy, vol. 6, pp. 128-134, Jun. 2009, doi: 10.1016/j.pdpdt.2009.07.002.

[10] Q. Sun et al., "Recent advances in terahertz technology for biomedical applications," Quant. Imag. Med. Surg., vol. 7, pp. 345-355, Jun. 2017, doi: 10.21037/qims.2017.06.02.

[11] U. A. Khan et al., "Broadband dielectric characterization of tumorous and nontumorous breast tissues," IEEE Trans. Microw. Theory Techn., vol. 55, no. 12 , pp. 2887-2893, Dec. 2007.

[12] T. Bowman, M. El-Shenawee, and L. K. Campbell, "Terahertz transmission vs reflection imaging and model-based characterization for excised breast carcinomas," Biomed. Opt. Express, vol. 7, no. 9, pp. 3756-3783, Sep. 2016, doi: 10.1364/BOE.7.003756.

[13] Z. Taylor et al., "THz medical imaging: In vivo hydration sensing," IEEE Trans. THz Sci. Technol., vol. 1, no. 1, pp. 201-219, Sep. 2011.

[14] A. Fitzgerald et al., "Terahertz pulsed imaging of human breast tumors," Radiology, vol. 239, no. 2, pp. 533-540, May 2006, doi: 10.1148/radiol.2392041315.

[15] C.Y. Jen and C. Richter, "Sample thickness measurement with THz-TDS: Resolution and implications," J. Infrared Millim. THz Waves, vol. 35, pp. 840-859, Oct. 2014, doi: 10.1007/s10762-014-0093-9.

[16] K. Su, Y.-C. Shen, and J. A. Zeitler, "Terahertz sensor for non-contact thickness and quality measurement of automobile paints of varying complexity," IEEE Trans. THz Sci. Technol., vol. 4, no. 4, pp. 432-439, Jul. 2014.

[17] S. Krimi et al., "Highly accurate thickness measurement of multi-layered automotive paints using terahertz technology," Appl. Phys. Lett., vol. 109, no. 2, Jul. 2016, Art. no. 021105, doi: 10.1063/1.4955407.

[18] J. van Mechelen, A. Kuzmenko, and H. Merbold, "Stratified dispersive model for material characterization using terahertz time-domain spectroscopy," Opt. Lett., vol. 39, no. 13, pp. 3853-3856, Jul. 2014, doi: 10.1364/OL.39.003853.

[19] Q. Cassar et al., "Iterative tree algorithm to evaluate terahertz signal contribution of specific optical paths within multilayered materials," IEEE Trans. THz Sci. Technol., vol. 9, no. 6, pp. 684-694, Nov. 2019

[20] P. Hillger, J. Grzyb, R. Jain, and U. R. Pfeiffer, "Terahertz imaging and sensing applications with silicon-based technologies," IEEE Trans. THz Sci. Technol., vol. 9, no. 1, pp. 1-19, Jan. 2019.

[21] K. Song, J. Kim, D. Kim, J. Yoo, and J.-S. Rieh, "A 300-GHz CMOS 7-by-7 detector array for optics-less $\mathrm{THz}$ imaging with scan-less target object," J. Infrared Millim. THz. Waves, vol. 41, pp. 202-214, Feb. 2020, doi: 10.1007/s10762-019-00664-3.

[22] D. Wang et al., " $240-\mathrm{GHz}$ four-channel power-tuning heterodyne sensing readout system with reflection and transmission measurements in a 130-nm SiGe BiCMOS technology," IEEE Trans. Microw. Theory Techn., vol. 67, no. 12, pp. 5296-5306, Dec. 2019.

[23] M. Bauer et al., "A high-sensitivity AlGaN/GaN HEMT terahertz detector with integrated broadband bow-tie antenna," IEEE Trans. THz Sci. Technol., vol. 9, no. 4, pp. 430-444, Jul. 2019.

[24] A. Arbabian, S. Callender, S. Kang, M. Rangwala, and A. M. Niknejad, "A $94 \mathrm{GHz}$ mm-wave-to-baseband pulsed-radar transceiver with applications in imaging and gesture recognition," IEEE J. Solid-State Circuits, vol. 48, no. 4, pp. 1055-1071, Apr. 2013.

[25] B. P. Ginsburg et al., "A $160 \mathrm{GHz}$ pulsed radar transceiver in $65 \mathrm{~nm}$ CMOS," IEEE J. Solid-State Circuits, vol. 49, no. 4, pp. 984-995, Apr. 2014.

[26] Y. Kawano et al., "RF chipset for impulse UWB radar using $0.13 \mu \mathrm{m}$ InPHEMT technology," IEEE Trans. Microw. Theory Techn., vol. 54, no. 12, pp. 4489-4497, Dec. 2006

[27] B. B. M. W. Badalawa and M. Fujishima, "60 GHz CMOS pulse generator," Electron. Lett., vol. 43, no. 2, pp. 100-102, Jan. 2007, doi: 10.1049/el:20073324. 
[28] C. Fang, C. L. Law, and J. Hwang, "High-voltage high-efficiency ultrawideband pulse synthesizer," IEEE Microw. Wireless Compon. Lett., vol. 20, no. 1, pp. 49-51, Jan. 2010.

[29] M. M. Assefzadeh and A. Babakhani, "Broadband oscillator-free THz pulse generation and radiation based on direct digital-to-impulse architecture," IEEE J. Solid-State Circuits, vol. 52, no. 11, pp. 2905-2919, Nov. 2017.

[30] M. Egard et al., "In0.53Ga0.47As RTD-MOSFET millimeter-wave wavelet generator," IEEE Electron Device Lett., vol. 33, no. 7, pp. 970-972, Jul. 2012.

[31] I. Vakili, L. Ohlsson, L.-E. Wernersson, and M. Gustafsson, "Time-domain system for millimeter-wave material characterization," IEEE Trans. Microw. Theory Techn., vol. 63, no. 9, pp. 2915-2922, Sep. 2015.

[32] S. N. Vainshtein et al., "Interferometrically enhanced sub-terahertz picosecond imaging utilizing a miniature collapsing-field-domain source," Appl. Phys. Lett., vol. 112, no. 19, May 2018, Art. no. 191104, doi: 10.1063/1.5022453.

[33] S. N. Vainshtein et al., "Collapsing-field-domain-based $200 \mathrm{GHz}$ solidstate source," Appl. Phys. Lett., vol. 115, no. 12, Sep. 2019, Art. no. 123501, doi: $10.1063 / 1.5091616$.

[34] R. M. Azzam and N. M. Bashara, Ellipsometry and Polarized Light. New York, NY, USA: North Holland, 1977.

[35] B. C. Q. Truong, H. D. Tuan, A. J. Fitzgerald, V. P. Wallace, and H. T. Nguyen, "A dielectric model of human breast tissue in terahertz regime," IEEE Trans. Biomed. Eng., vol. 62, no. 2, pp. 699-707, Feb. 2015.

[36] E. A. J. Marcatili and R. A. Schmeltzer, "Hollow metallic and dielectric waveguides for long distance optical transmission and lasers," Bell Syst. Techn. J., vol. 43, pp. 1783-1809, Jul. 1964, doi: 10.1002/j.15387305.1964.tb04108.x.

[37] N. Fuse and T. Fukuchi, "Estimation of surface scattering effect on terahertz non-contact detection of underfilm corrosion," IEEJ Trans. Fundam. Mater, vol. 137, pp. 158-164, Mar. 2017, doi: 10.1541/ieejfms.137.158.

[38] N. Fuse, T. Fukuchi, T. Takahashi, M. Mizuno, and K. Fukunaga, "Evaluation of applicability of noncontact analysis methods to detect rust regions in coated steel plates," IEEE Trans. THz Sci. Technol., vol. 2, no. 2, pp. 242-249, Mar. 2012.

[39] Y.-S. Jin, G.-J. Kim, and S.-G. Jeon, "Terahertz dielectric properties of polymers," J. Korean Phys. Soc., vol. 49, no. 2, pp. 513-517, 2006.

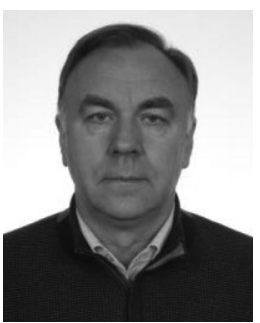

Valeri A. Mikhnev (Senior Member, IEEE) graduated from the Faculty of Radio Physics, Belarusian State University, Minsk, Belarus, in 1979, and the Ph.D. and D.Sc. degrees from the Institute of Applied Physics, National Academy of Sciences of Belarus, Minsk, Belarus, in 1986 and 2003, respectively.

During 1979-2011, he was a Researcher, then the Head of the Laboratory of Microwave Methods for Nondestructive Testing with the Institute of Applied Physics, National Academy of Sciences of Belarus. During 2011-2013, he was a Researcher with Aalto University, Espoo, Finland, where he studied subsurface radar signal processing. During 2014-2019, he was a Visiting Scientist with the University of Oulu, Oulu, Finland, where he was involved in the development of pulsed terahertz imaging systems. His current research interests include millimeter-wave imaging and material characterization.

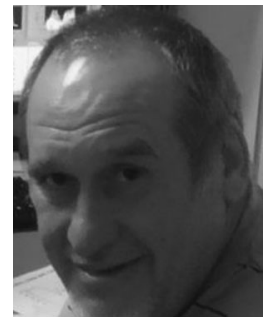

Sergey N. Vainshtein was born in Kostroma, USSR, in 1955. He received the M.Sc. degree (with honors) in physics from Leningrad Polytechnic Institute, Leningrad, USSR, in 1978, and the Ph.D. degree in semiconductor physics from the A. F. Ioffe Institute of Physics and Technology, St. Petersburg, Russia, in 1987.

From 1980 to 2007, he was with the A. F. Ioffe Institute of Physics and Technology. Since 1995, he has been a Senior Scientist with CAS Group, ITEE Department, University of Oulu, Oulu, Finland. His current research interests include pulsed all-electronic THz emitters, high-speed semiconductor devices based on avalanche switching, picosecond kinetics of laser diodes and light emitting diodes, and original pulsing circuits for their pumping.
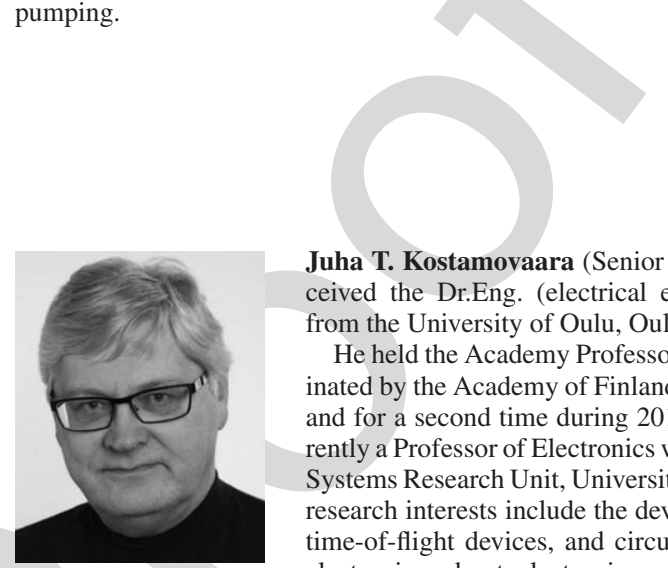

Juha T. Kostamovaara (Senior Member, IEEE) received the Dr.Eng. (electrical engineering) degree from the University of Oulu, Oulu, Finland, in 1987.

He held the Academy Professorship Position nominated by the Academy of Finland during 2006-2011 and for a second time during 2012-2017. He is currently a Professor of Electronics with the Circuits and Systems Research Unit, University of Oulu. His main research interests include the development of pulsed time-of-flight devices, and circuits and systems for electronic and optoelectronic measurements. 
GENERAL INSTRUCTION

- Authors: Carefully check the page proofs (and coordinate with all authors); additional changes or updates WILL NOT be accepted after the article is published online/print in its final form. Please check author names and affiliations, funding, as well as the overall article for any errors prior to sending in your author proof corrections.

- Authors: We cannot accept new source files as corrections for your article. If possible, please annotate the PDF proof we have sent you with your corrections and upload it via the Author Gateway. Alternatively, you may send us your corrections in list format. You may also upload revised graphics via the Author Gateway.

QUERY

Q1. Author: Please confirm or add details for any funding or financial support for the research of this article. 


\title{
Time-Domain Terahertz Imaging of Layered Dielectric Structures With Interferometry-Enhanced Sensitivity
}

\author{
Valeri A. Mikhnev ${ }^{\circledR}$, Senior Member, IEEE, Sergey N. Vainshtein,

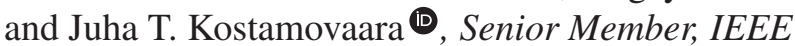

\begin{abstract}
This article presents a time-domain imaging technique for layered dielectric slabs using a solid-state wavelet generator with subterahertz carrier frequency. The technique utilizes the dual nature of a wavelet, i.e., both the applicability of time-offlight measurements and the ability of wavelets to interfere in thin dielectric layers at a carrier frequency that is preserved in spite of the ultrawideband character of the signal. This results in a very high sensitivity of the time delay of the resultant pulse to variations in the effective thickness (thickness $\times$ refractive index) of the dielectric layer. It is shown using a plane-wave analysis of the pulse propagation that under certain conditions, this sensitivity enhancement can reach an order of magnitude. The experimental setup for the reflection-mode operation is described and its performance in the discrimination of healthy and malignant tissues and in the detection of corrosion under paint is demonstrated.
\end{abstract}

Index Terms-Biomedical imaging, interferometry, layered dielectric structures, nondestructive testing, solid-state terahertz (THz) pulsed source, $\mathrm{THz}$ active imaging.

$\mathbf{T}$ HANKS to the transparency of most nonconductive materials in the subterahertz $(\mathrm{THz})$ frequency range and good spatial resolution, $\mathrm{THz}$ waves have found a wide range of applications in imaging and sensing in recent years [1]-[3]. This imaging technology has demonstrated promising results in nondestructive inspection [4], [5], security imaging applications [6], food safety testing [7], etc.

The nonionizing energies of sub- $\mathrm{THz}$ photons also give rise to multiple applications in biomedical imaging [8]-[10]. THz waves are sensitive to water content and thus, its variation within tissue samples can be utilized for medical diagnostics. The contrast in dielectric properties between healthy and malicious tissues [11]-[13] caused by water content variation has been used for the detection of skin, colon, and breast cancer, even

Manuscript received February 14, 2020; revised May 14, 2020; accepted June 5, 2020. This work was supported by the Academy of Finland under Contract 307362 (Center of Excellence in Laser Scanning Research) and Contract 310152. (Corresponding author: Valeri A. Mikhnev.)

The authors are with the Faculty of Information Technology and Electrical Engineering, Oulu University, FIN-90014 Oulu, Finland (e-mail: v.mikhnev@ieee.org; sergey.vaynshteyn@oulu.fi; juha.kostamovaara@oulu.fi).

Color versions of one or more of the figures in this article are available online at https://ieeexplore.ieee.org.

Digital Object Identifier 10.1109/TTHZ.2020.3003500 though the difference in hydration is not the only mechanism responsible for this contrast [14].

Contactless $\mathrm{THz}$ measurement technology has also proven to be highly suitable for industrial applications in the inspection of layered nonmetallic materials, such as plastic, resin, ceramics, and paint coatings. Accurate evaluation of the refractive index and/or thickness of every layer can be performed by using a variety of time-domain and frequency-domain techniques. Relatively thick layers with clear separation of $\mathrm{THz}$ pulses reflected from the layer interfaces can be characterized by simple time-of-flight and phase-slope methods [5], [15], and even better results have been obtained with model-based optimization routines aimed at the best fit between numerical and experimental data [16]-[18]. In very thin stratified structures, however, the received $\mathrm{THz}$ waveforms become difficult to interpret due to overlapping of the partial pulses reflected from the layer interfaces. In this case, extraction of the parameters of interest requires more sophisticated methods based on the solution of an inverse problem or iterative tree algorithm [19].

Most of these advances have been enabled by the discovery of the $\mathrm{THz}$ time-domain spectroscopy method, but in spite of its success, this method has well-known drawbacks, such as the bulky and costly laser-based instrumentation, low output power, and a rather long acquisition time.

Considerable developments have been made in the past decade to both continuous-wave [20]-[23] and pulsed [24]-[33] solid-state components that can be expected to drive the system costs down and promote applications of the THz-time-domain imaging (TDI) technology. Ultrawideband (UWB) millimeterwave and sub-THz pulse generators that are well suited for high-frame-rate imaging are also popular in radar and wireless communications, where a variety of carrier-based and noncarrier-based architectures have been proposed. In the carrier-based scheme, a high-frequency carrier is modulated with a short pulse, producing a high-frequency wavelet, typically with a Gaussian envelope [24]-[25]. A higher power efficiency is offered by noncarrier-based wavelet generators that are built using various schemes such as bandpass shaping of ultrashort pulses [26], edge-combining [27] and pulse-combining [28] methods, and direct digital-to-impulse conversion [29], to name just a few. Gaussian-envelope wavelets can also be generated by 
circuits based on a resonant tunneling diode (RTD) [30]. A timedomain system for material characterization employing this type of pulsed source has already been reported [31]. As these solid-state devices are fabricated by planar microlithographic technology, they can be scaled up to arrays with integrated antennas suitable for multipixel imaging [21], [22].

Another generator of short sub-THz pulses is based on the concept of collapsing-field domains arising in a GaAs avalanche transistor under certain conditions [32], [33]. This produces a train of Gaussian-envelope wavelets with a peak power of a few milliwatts, a center frequency ranging from 130 to $200 \mathrm{GHz}$, depending on the transistor design, and a duration of 40-100 ps. The single-shot jitter of this source is as low as $\sim 1 \mathrm{ps}$, which makes it well suited for applications requiring accurate measurements of the time of flight. It was also noticed that there exists a remarkable interference of the wavelets reflected from the interfaces of layered dielectric structures that considerably affects the time of flight of the resultant pulse. The discovery of interferometric enhancement of the sensitivity was found to be promising for TDI applications.

This article will present a comprehensive analysis of the transmission and reflection of a sub-THz pulse with a Gaussian envelope in multilayered dielectric structures, showing that in a properly configured measurement system, the time delay of the received pulse is very sensitive to the effective thickness of the layers in the stratified structure. The variation in the time delay may be an order of magnitude higher than variation of the intrinsic time delay of the electromagnetic wave in the same dielectric material. Combined with the source jitter of $\sim 1$ ps and pulse averaging, the interferometric enhancement technique provides time-of-flight measurements with subpicosecond resolution. Consequently, this measurement approach based on a low-cost all-electronic solid-state sub-THz source with a bandwidth of about $30 \mathrm{GHz}$ [32] may prove competitive in some TDI applications with bulky and costly THz-TDS systems having a bandwidth of a few THz. The performance of the interferometric enhancement technique has been demonstrated in several simulated and experimental examples, including such complicated tasks as the discrimination of healthy and cancerous areas in freshly excised tissue slices. It has also been applied successfully for the detection of corrosion in metal under paint.

The article is organized as follows. Section II addresses the technique of interferometric enhancement, its conditions and limitations. A reflection-mode measurement setup including the pulsed sub-THz source, lenses, and a Schottky detector mounted in a common beam guide is presented in Section III. The experimental results are presented in Section IV, and Section V concludes this article along with proposals for future work.

\section{DESCRIPTION OF THE METHOD}

The proposed TDI technique is based on time-of-flight measurements of a Gaussian-envelope wavelet reflected or transmitted through a multilayered dielectric slab at normal incidence (see Fig. 1).

The corresponding waveforms can be determined as a convolution of the incident signal waveform with the impulse transfer

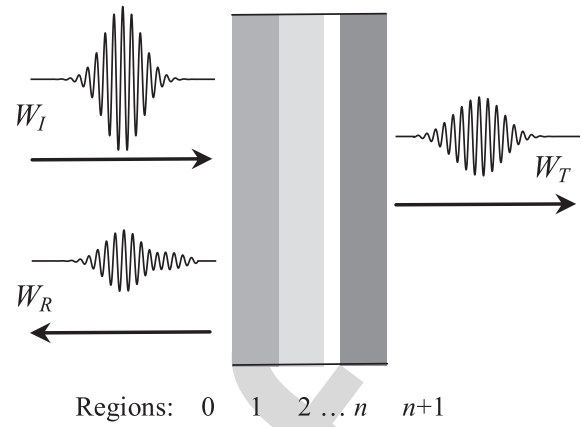

Fig. 1. Geometry of the problem. $W_{I}, W_{R}$, and $W_{T}$ represent incident, reflected, and transmitted wavelets, respectively.

functions of the slab. In the case of a multilayered slab, it is convenient to find its transfer function in the frequency domain with subsequent use of the convolution theorem. Reflected and transmitted wavelets $W_{R}$ and $W_{T}$ can then be calculated using the formulas

$$
\begin{aligned}
& W_{R}(t)=\Phi^{-1}\left\{\Phi\left[W_{I}(t)\right] \cdot R(f)\right\} \\
& W_{T}(t)=\Phi^{-1}\left\{\Phi\left[W_{I}(t)\right] \cdot T(f)\right\}
\end{aligned}
$$

where $W_{I}(t)$ is the incident wavelet, $\Phi$ and $\Phi^{-1}$ are direct and inverse Fourier transforms, and $R(f), T(f)$ are the reflection and transmission coefficients as functions of the frequency $f$.

The functions $R(f)$ and $T(f)$ can both be derived using a concept of scattering transfer matrices that are cascadable and thus, make it easy to deal with multiple layers [34]. Accordingly, a $n$-layer slab is described in the plane-wave case by the product of transfer matrices for the dielectric interfaces and the homogeneous layers between them

$$
\mathbf{A}=\mathbf{I}_{0,1} \mathbf{L}_{1} \mathbf{I}_{1,2} \mathbf{L}_{2} \mathbf{I}_{2,3} \ldots \mathbf{L}_{n} \mathbf{I}_{n,(n+1)}
$$

where $n$ is the number of layers

$$
\begin{aligned}
\mathbf{I}_{(i-1), i} & =\frac{1}{1+r_{(i-1), i}}\left[\begin{array}{cc}
1 & r_{(i-1), i} \\
r_{(i-1), i} & 1
\end{array}\right], i \\
& =1,2, \ldots,(n+1)
\end{aligned}
$$

is the transfer matrix of the interface between regions $(i-1)$ and $i$, and

$$
\mathbf{L}_{i}=\left(\begin{array}{cc}
\exp \left(j \beta_{i}\right) & 0 \\
0 & \exp \left(-j \beta_{i}\right)
\end{array}\right), i=1,2, \ldots, n
$$

is the transfer matrix of the layer $i$. Assuming that the dielectrics of interest are nonmagnetic, the Fresnel coefficients $r_{(i-1), i}$ and the propagation constants $\beta_{i}$ are related to the complex dielectric permittivities of the layers $\varepsilon_{i}$ and their thicknesses $d_{i}$ by

$$
r_{(i-1), i}=\frac{\sqrt{\varepsilon_{\iota-1}}-\sqrt{\varepsilon_{i}}}{\sqrt{\varepsilon_{\iota-1}}+\sqrt{\varepsilon_{i}}} \quad \beta_{i}=\frac{2 \pi f}{c} d_{i} \sqrt{\varepsilon_{i}}
$$

where $c$ is the velocity of light.

Once the complete matrix $\mathbf{A}$ of the multilayered structure is calculated, the frequency-domain transfer functions $R(f)$ and $T(f)$ required in (1) can be obtained as

$$
R(f)=A_{21} / A_{11} \quad T(f)=1 / A_{11}
$$




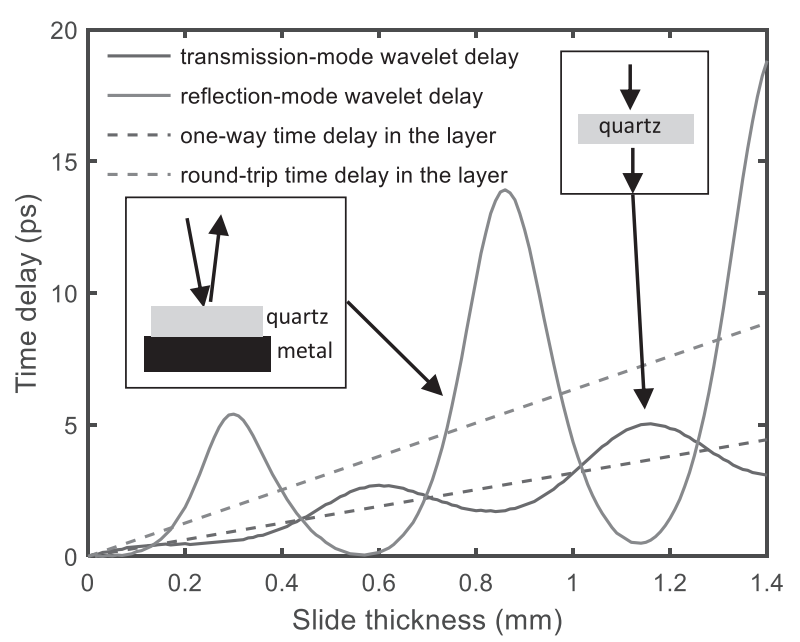

Fig. 2. Calculated time delay of a Gaussian-envelope wavelet (center frequency $135 \mathrm{GHz}$, FWHM 50 ps) transmitted through a quartz layer with a dielectric permittivity $\varepsilon=3.8$ (blue line, geometry shown in the right inset) and reflected from a metal-backed quartz layer (magenta line, geometry is shown in the left inset) versus the layer thickness. The dashed blue and dashed magenta lines show the time delays in the layer as they would be in the absence of interference.

and the final results for the transmitted and reflected wavelets are now accessible from (1).

For transmission-mode modeling, both of the half-spaces (regions 0 and $(n+1)$ in Fig. 1) confining the multilayered slab are assumed to be air. The reflection mode with a metal-backed layer is realized by setting $r_{n,(n+1)}=-1$, implying that the region $(n+1)$ is metal, and then, $W_{T}$ disappears.

In order to obtain the time-delay values comparable with the experimental data, one has to subtract from the propagation time of the wavelet obtained from (1) the propagation time in an air layer of the same thickness as the dielectric sample. This is a consequence of the conventional calibration of the time-delay measurement needed to subtract the time delay in the sensing head, connecting cables, etc. As the initial time delay measured in the experimental setup in the absence of a sample also includes the time delay in the air layer occupied by the dielectric sample at that time, correct comparison requires removing the corresponding time delay from the simulated results, too. In the transmission mode, this means subtraction of a term $\Delta t=\sum d_{i} / c$, while in the reflection mode for a metal-backed layer, the doubled term $2 \Delta t$ needs to be subtracted due to the round trip of the electromagnetic wave in the layer.

The method described above suits well to obtain transmitted and reflected pulses in many practical scenarios with multilayered structures in biomedical imaging and nondestructive testing. Consider first the case of a single layer as far as both its transmission and reflection geometry is concerned. The time delay calculated for the wavelets transmitted through a single dielectric layer and reflected from the same layer placed on a metal substrate is shown in Fig. 2. A Gaussian wavelet with a carrier frequency of $135 \mathrm{GHz}$ and a full width at half-maximum (FWHM) of 50 ps has been adopted here as $W_{I}$.

As seen in Fig. 2, the time delay of both transmitted and reflected wavelets as a function of the layer thickness resembles an interferogram despite the fact that the incident wavelet fulfills the definition of a UWB signal. One must keep in mind, however, that this interferogram is unusual, since the time delay rather than the amplitude is plotted here.

The observed behavior of the time delay can be explained by the interference of the wavelets reflected from the layer interfaces. Thus, in the case of reflection-mode operation with a metal-backed layer, the wavelet reflected from the first interface of the layer overlaps with the leading edge of the wavelet reflected from the metal surface that arrives later.

These wavelets can interfere constructively or destructively, depending on the electrical thickness of the layer. Destructive interference suppresses the leading edge of the resultant pulse that appears as its additional delay, but when the interference is constructive, the leading edge swells up so that the resultant pulse appears to be not delayed at all, e.g., at layer thicknesses of 0.57 and $1.14 \mathrm{~mm}$ in Fig. 2. Incidentally, the specified thicknesses correspond exactly to the half-wavelength and one-wavelength quartz layers at a frequency of $135 \mathrm{GHz}$, respectively. This is clear evidence of the interference nature of the graph in Fig. 2. Alternatively, the maxima of the time delay in the reflection mode may appear when the effective thickness of the layer is equal to an odd number of quarter-wavelengths.

The oscillations in the time delay of the Gaussian wavelet versus the electrical thickness of the dielectric layer can be used for the imaging of electrically thin dielectric structures. To this end, the operating point should be chosen on one of the slopes of the "interferogram" in Fig. 2. Then, small variations in either the refractive index or the thickness of the layer will result in pronounced variations in the time delay.

As the oscillations in the time delay are much stronger in the reflection mode, this mode is preferred in further considerations because of the higher expected sensitivity. Nevertheless, the transmission mode can be useful, too. For instance, a high spatial resolution can be achieved in the transmission mode by using a focused beam and a detector with a small aperture. There is also some gain in sensitivity in this mode, although lower than in the reflection mode.

The time delay in the resultant pulse has been determined in the above calculations from the position of its maximum. Detection of the position of the rising edge of the pulse is preferable in the experimental studies for the sake of better measurement accuracy, but in this case, the Gaussian shape of the received wavelet should be preserved. The wavelet envelopes were calculated for a set of maxima [see Fig. 3(a)] and minima [see Fig. 3(b)] in the reflection mode using the geometry of the left inset in Fig. 2 and the same characteristics of the probing wavelet. For clarity, only two maxima and two minima of the time delay are shown in Fig. 2, whereas in Fig. 3(a), the envelopes of the first four maxima are plotted (the corresponding quartz thicknesses are $0.28,0.85,1.42$, and $1.99 \mathrm{~mm}$ ).

The time scale in Fig. 3 is selected so that the apex of the probing wavelet corresponds to zero time. It is seen in Fig. 3(a) that the wavelets of the first two maxima preserve their Gaussian shape well. At the next maxima, the pulse shape is distorted and widened, although it suits quite well for time-of-flight measurements. 


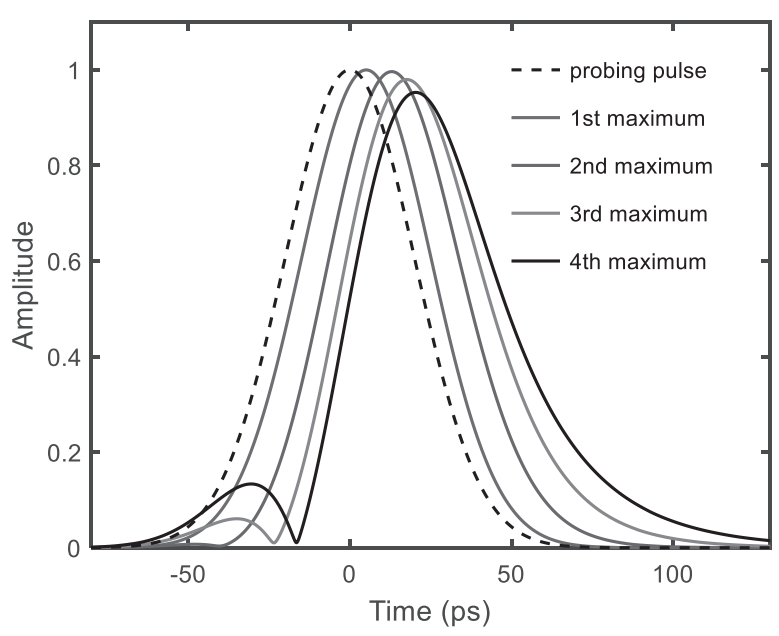

(a)

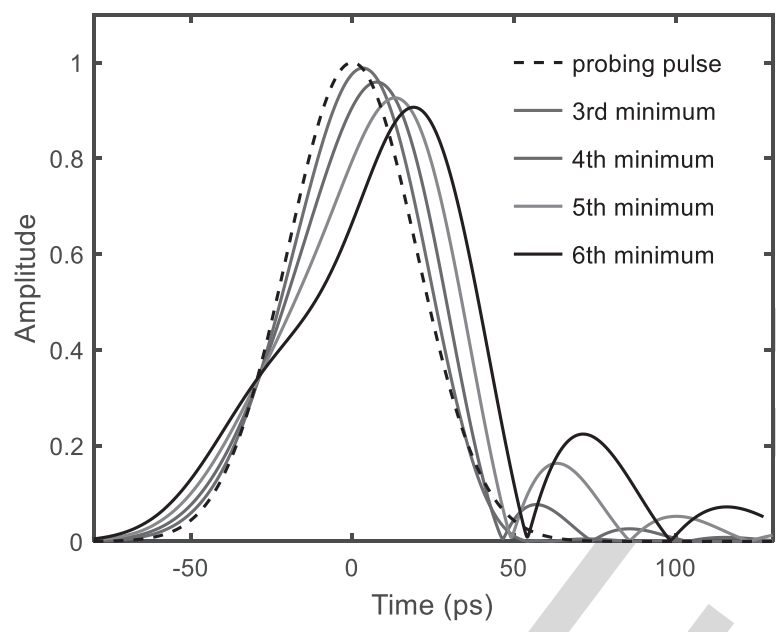

(b)

Fig. 3. Shapes of the envelopes of received wavelets in the reflection mode of operation using the geometry shown in the left inset in Fig. 2 and the same characteristics of the probing wavelet. (a) Shapes corresponding to a set of the first four maxima of the time delay (thicknesses of quartz substrate are 0.28 , $0.85,1.42$, and $1.99 \mathrm{~mm}$ ). (b) Shapes corresponding to a set of minima of the time delay numbered 3-6 (corresponding quartz thicknesses are 1.71, 2.28, 2.85, and $3.42 \mathrm{~mm}$ ).
In Fig. 3(b), the shapes of the received pulses for the minima numbered 3-6 (with corresponding quartz thicknesses of 1.71, $2.28,2.85$, and $3.42 \mathrm{~mm}$ ) are plotted. The wavelet shapes for the first and second minima (layer thicknesses 0.57 and $1.14 \mathrm{~mm}$ ) are not shown here as they are graphically indistinguishable from the probing wavelet. As in Fig. 3(a), the pulses of the first minima in Fig. 3(b) keep their Gaussian shapes, being almost exact replicas of the probing wavelet. At greater thickness of the substrate, however, the shape of the received pulse is rapidly distorted and the slope of the time-delay interferogram does not increase any longer. Hence, for implementation of the method, the dielectric substrate must be thick enough to achieve better sensitivity, while at the same time avoiding any marked deviation of the received pulse from the Gaussian shape.

It is intuitively expected from Fig. 2 that the areas of high sensitivity to a thin dielectric layer placed over the metal-backed

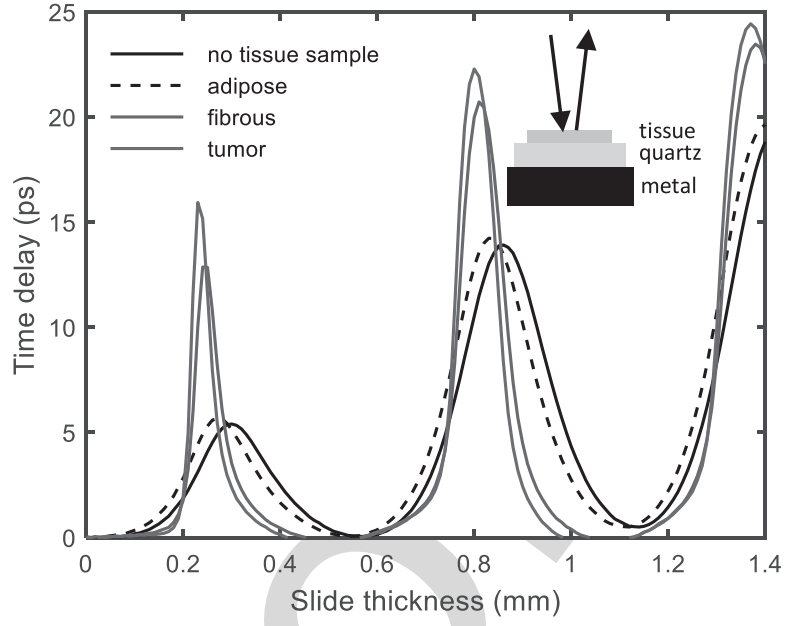

Fig. 4. Time delay of the Gaussian-envelope wavelet (center frequency $135 \mathrm{GHz}, \mathrm{FWHM} 50 \mathrm{ps}$ ) versus thickness of the quartz slide calculated for the "tissue-quartz-metal" structure in the reflection mode. The tissue slice thickness is $50 \mu \mathrm{m}$, and the dielectric permittivities of the three tissue types (adipose, fibrous, and tumorous) are taken to be $\varepsilon_{a}=2.4-\mathrm{j} 1.0 ; \varepsilon_{f}=3.6-\mathrm{j} 5.3$; $\varepsilon_{t}=4.4-\mathrm{j} 6.2$, respectively. Inset: Simulation model.

slide will correspond to both slopes in the time-delay curve. To verify this, we calculated the time-delay variations for three types of fresh tissue slices-adipose, fibrous, and tumorous (see Fig. 4), the dielectric permittivity data for which are taken from [35]. The simulation model is shown in the inset in Fig. 4.

It is seen from the simulation results that the sensitivity of the method to the dielectric properties of a thin layer does indeed increase considerably, so that the additional round-trip propagation time of the electromagnetic wave in the 50- $\mu$ m layer with a refractive index of the fibrous tissue of $n_{f}=\operatorname{Re} \sqrt{\varepsilon_{f}} \cong 2.25$ can be estimated to be $2 d\left(n_{f}-1\right) \cong 0.4 \mathrm{ps}$, while at some points in Fig. 4, the corresponding time-delay difference reaches 8-9 ps. This constitutes the principle of interferometric enhancement for pulsed time-domain imaging. One must keep in mind that the results obtained here correspond to the parameters of pure tissues whereas in real slices, the tissue types may be intermingled.

The slice thickness should be chosen as a tradeoff between sensitivity and the interference conditions. Too thin a tissue sample will make a low contribution to the reflected signal, while too thick a sample will suppress the effect of the interference. For the tissues considered here and the signal parameters specified in Fig. 4, the optimal range of slice thickness should be about 50 to $100 \mu \mathrm{m}$.

The most complicated case in breast cancer imaging is the differentiation between fibrous and cancerous tissues, as their dielectric properties are very similar [35]. The estimated round-trip propagation times in $50 \mu \mathrm{m}$ slices of those tissues yield a difference of only $\sim 0.1 \mathrm{ps}$, which is very challenging even for femtosecond laser-based pulsed $\mathrm{THz}$ imaging, but interferometry-enhanced TDI can increase the difference to 1-2 ps (see Fig. 4) if the thickness of the quartz slide is selected properly.

Finally, we should consider the influence of the wavelet center frequency and the dielectric permittivity of the slide on the efficiency of interferometric enhancement (see Fig. 5). 


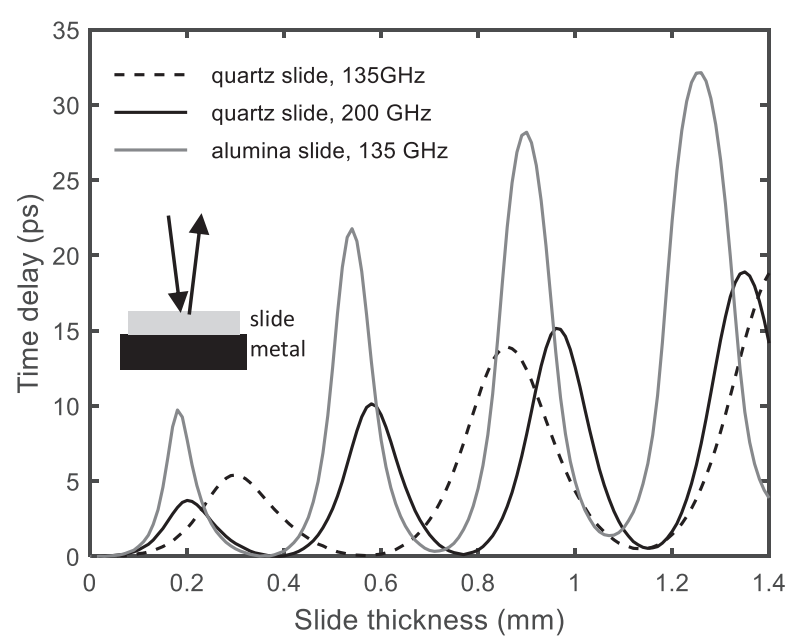

Fig. 5. Calculated time delay of the Gaussian-envelope wavelets versus slide thickness in the reflection mode (see inset) for different slides and center frequency of the wavelet: Quartz slide and frequency of $135 \mathrm{GHz}$ (dashed black); alumina slide and frequency of $135 \mathrm{GHz}$ (magenta); quartz slide and frequency of $200 \mathrm{GHz}$ (solid black).

As the center frequency of the wavelet increases (e.g., by using a 200-GHz pulsed source [33]), more oscillations of the time delay occur in the same interval of thickness that is natural for the interferometry. Consequently, the slopes of the "interferogram" become steeper, leading to a higher sensitivity. A similar effect can also be achieved by replacing quartz with a dielectric having a larger refractive index, e.g., alumina, with dielectric permittivity $\varepsilon=9.6$ (see Fig. 5, magenta line). The use of a high-permittivity substrate is not reasonable in this arrangement, however, despite the attractive steepness of the interference curve. The problem is that the amplitudes of the interfering pulses are comparable in this case, and this may lead to disintegration of the resultant wavelet and thus, to jumps in the measured time delay. Consequently, both the type and thickness of the slide and the frequency of operation must be optimized for any practical scenario by means of preliminary simulations, as in Fig. 4.

The operating point on the inclined section of the time-delay interferogram can also be selected experimentally. To this end, thin quartz slides with a thickness of $0.2-0.3 \mathrm{~mm}$ can be added to the main quartz slide and thus, the substrate thickness corresponding to the highest sensitivity in the inclined section of the time-delay interferogram can be estimated. In addition, it should be kept in mind that there exists an intrinsic limitation to the range of the time delay, which cannot exceed approximately half-FWHM, because the interfering wavelets must overlap well in order to create the required effect.

\section{EXPERIMENTAL SETUP}

The pulsed sub-THz source with an on-chip semicircular bowtie antenna [32] was fabricated on a $100-\mu \mathrm{m}$ GaAs substrate using state-of-the-art heterojunction bipolar transistor (HBT) technology with some modifications. The fabricated chip was mounted on the flat side of a hyperhemispherical high-resistivity

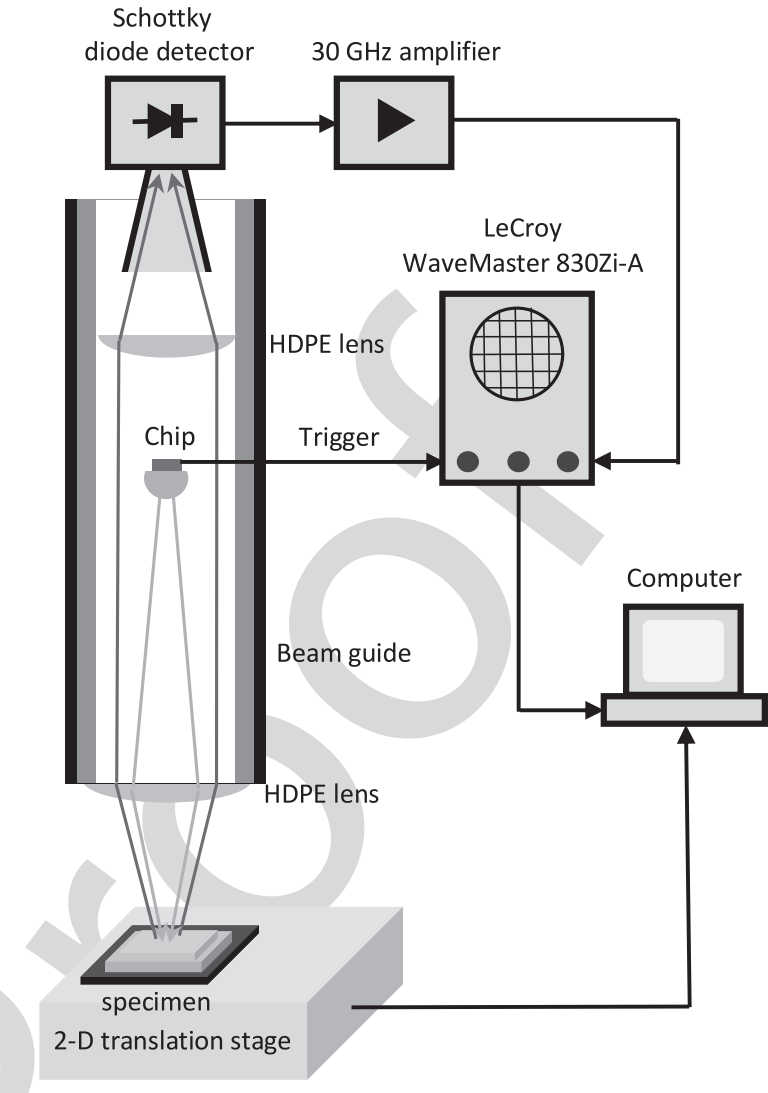

Fig. 6. Reflection-mode experimental setup. Propagation of the incident wave is shown schematically by blue lines, and that of the reflected wave is shown in red. The specimen under test (yellow) is placed on a metal-backed quartz slide (blue).

silicon lens with a diameter of $4 \mathrm{~mm}$ for efficient coupling of the radiation to the free space. The output power of the emitter was measured using a special setup consisting of a large elliptical mirror, a Golay cell, and a set of metal-mesh quasi-optical bandpass filters. The typical peak output power was $\sim 1 \mathrm{~mW}$. The emitter was then placed in the center of a hollow dielectric beam guide supporting a Gaussian beam at frequencies above $100 \mathrm{GHz}$ with low attenuation [36]. A simplified functional scheme of the experimental setup is shown in Fig. 6 .

The pulse is emitted by the chip through the silicon lens and propagates along the beam guide with a diameter of $20 \mathrm{~mm}$. It is then focused by a high-density polyethylene (HDPE) lens (diameter $25.4 \mathrm{~mm}$, focal length $25 \mathrm{~mm}$ ) onto a layered structure including a metal substrate, a quartz slide, and a specimen of the material under test. The reflected wavelet propagates through the HDPE lens in the opposite direction and after some shaping by the beam guide, circumvents the emitter. In the end, it is collected by a waveguide horn antenna with attached zero-bias Schottky detector (Virginia Diodes, 110-170 GHz) matched well at the wavelet center frequency of about $135 \mathrm{GHz}$. To enhance the amplitude of the received signal, another HDPE lens is placed in front of the waveguide horn at a distance that ensures maximum signal transfer from the beamguide mode to the detector.

The detected wavelet envelopes were recorded using a LeCroy WaveMaster 830Zi-A oscilloscope. In the future, we plan to 
replace the oscilloscope with an ultrafast time-to-digital converter, but this is a difficult problem as the oscilloscope is triggered from a current pulse formed in the source supply circuit during avalanche switching simultaneously with the sub- $\mathrm{THz}$ emission, and thus, the single-shot jitter is $\sim 1$ ps. This nevertheless promises high-precision time-of-flight measurements, especially with the use of averaging, although it also imposes strict requirements on the time-to-digital converter. The specimen is mounted on a computer-controlled motorized translation stage and scanned two-dimensionally during data acquisition to form the image.

The spatial resolution was evaluated by a knife-edge method and is about $5 \mathrm{~mm}$, which agrees well with the diffraction limit at the frequency of operation.

The reflection-mode setup design presented here differs from the conventional schemes that employ either a beam splitter or a scheme under oblique incidence [9], [12], [14]. Although there are some losses in our system related to high divergence of the incident beam and partial shielding of the reflected wave by the emitter lens, the reflected signal was still found to be larger than in the usual design based on a beam splitter. Besides, the design shown in Fig. 6 is very compact and robust, as all the critical components are placed in a common metal tube of diameter $40 \mathrm{~mm}$ embracing the hollow dielectric beam guide. This makes it attractive for future practical applications.

\section{EXPERIMENTAL RESULTS}

For experimental verification of the effect of interferometric enhancement in the reflection mode, we prepared a sample of polyethylene terephtalate (PET) with a steplike thickness profile mounted on a metal substrate. PET was chosen due to the relative simplicity of preparing multiple layers of different thicknesses. In our specimen, they varied in thickness between 0 and $1.2 \mathrm{~mm}$ in steps of $0.2 \mathrm{~mm}$. The width of each layer was about 7-8 $\mathrm{mm}$, which is a little larger than the spatial resolution of the experimental setup. Strips of clean metal were left at both ends of the fabricated specimen for performing calibration of the time delay. The time-delay measurements were also performed for several available quartz slides with thicknesses in the range $0.8-1.3 \mathrm{~mm}$, and their results are plotted in Fig. 7 along with the curves obtained by numerical simulations for the center frequency of $135 \mathrm{GHz}, \mathrm{FWHM} 40 \mathrm{ps}$, and the dielectric permittivity of PET $\varepsilon=2.56$ and quartz $\varepsilon=3.8$.

The oscillating behavior of the time delay of the reflected pulse depending on the thickness of the metal-backed layer was demonstrated experimentally for two types of dielectrics. The experimental points agree reasonably well with the results of the numerical simulations, the differences being in most cases about 1-1.5 ps, which is close to the values for the jitter and drift of the pulsed source and digital oscilloscope employed in the experimental setup.

The high sensitivity and picosecond resolution of the time-offlight measurements look promising with respect to resolving the challenging problems of biomedical diagnostics such as differentiation between healthy and malignant tissues. Due to the high absorption of the sub-THz signal in biological materials,

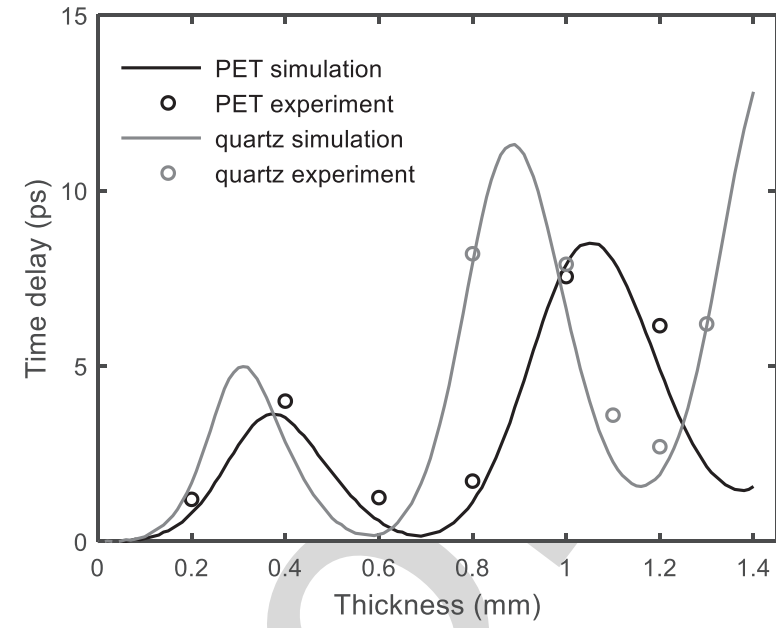

Fig. 7. Time delay of the Gaussian-envelope wavelets (center frequency $135 \mathrm{GHz}, \mathrm{FWHM} 40 \mathrm{ps}$ ) reflected from PET and quartz layers of different thickness. The experimental results are shown by circles. The results of the numerical simulations are also given for comparison (solid lines).

sample preparation must rely either on very thin tissue, with a loss of sensitivity, or on dehydration of the sample through formalin fixing and embedding in paraffin in order to maintain a reasonable transmitted signal. If the pathological analysis of a paraffin-embedded sample shows positive margins of the cancerous area, a second operation will be required, which is highly undesirable for many reasons, whereas if the decision could be made intraoperatively by analyzing freshly excised tissue slices, then the malignant tumor could be removed in a single operation. The results of our first experiments with the breast cancer tissue samples are presented in Fig. 8.

First, the tissue slices provided by Oulu University Hospital were measured in transmission mode, i.e., a 300- $\mu$ m thick slice was mounted on a quartz slide of thickness $1.2 \mathrm{~mm}$. The contrast of the obtained images was very poor for both amplitude and time-of-flight data and thus, no clear correlation with the histology could be found.

The images were then built up using the reflection-mode setup shown in Fig. 6 and much thinner slices, of thickness only $100 \mu \mathrm{m}$. The contrast in time delay measured over the tissue slice increased to $5 \mathrm{ps}$ when the $1.2-\mathrm{mm}$ quartz slide was used [see Fig. 8(b)]. This corresponded to the ascending branch of the "interferogram" in Fig. 7. In another experiment, the operating point was selected on the descending branch of the curve in Fig. 7 by using a quartz slide of thickness $1.0 \mathrm{~mm}$. As a result, the contrast with the time delay recorded over the same tissue slice increased to 13 ps [see Fig. 8(c)].

The area of a pronounced deviation of the time delay from the background values in Fig. 8(b) and (c) corresponds satisfactorily to the histological position of tumorous area [see Fig. 8(a)], although the spatial resolution needs to be improved. We expect that the next designs employing 200-300 GHz sources [33] will solve this problem.

Another promising application for the interferometric enhancement technique is the detection of corrosion under paint. Identifying corrosion before it becomes visible can help to 


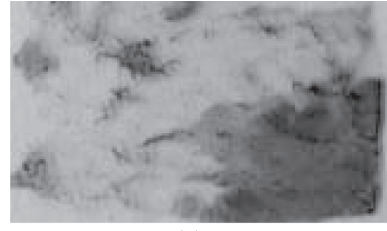

(a)

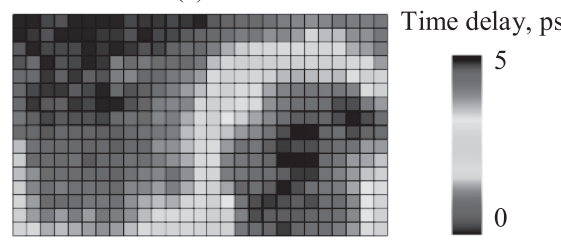

(b)

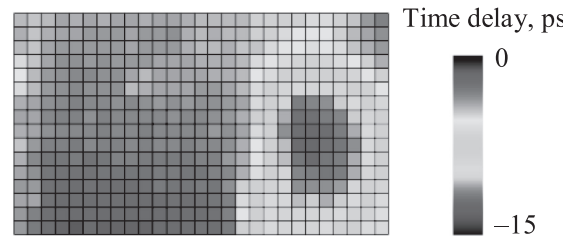

(c)

Fig. 8. Images of successive slices of the same fresh-frozen breast tumor tissue. (a) Histology, where adipose tissue is transparent, fibrous tissue is pink and intermingled with adipose, and tumor tissue is violet-pink. (b) Time-of-flight reflection-mode image obtained with a $100-\mu \mathrm{m}$ slice placed on a $1.2-\mathrm{mm}$ quartz slide. (c) Time-of-flight reflection-mode image obtained with the $100-\mu \mathrm{m}$ slice placed on a 1.0-mm quartz slide. The time-delay values in (b) and (c) mean a delay difference related to a selected operating point. The size of the grid cell in (b) and (c) is of $1 \mathrm{~mm} \times 1 \mathrm{~mm}$.

avoid potential structural problems and minimize repair costs. Corrosion causes roughness of a metal surface that in turn results in marked scattering of electromagnetic waves of short wavelengths. Consequently, corroded areas can be detected as areas of lower reflectivity in the sub-THz reflection image obtained at normal incidence [37], [38].

The applicability of the interferometric enhancement technique to the detection of hidden corrosion has been examined using the experimental setup shown in Fig. 6 and a specially processed specimen of homogeneously rusted steel in which the rust had been partially removed to form alternating strips of rusty and clean metal approximately $15 \mathrm{~mm}$ in width [see Fig. 9(a)].

The specimen was placed on the translation stage as shown in Fig. 6 and scanned twice in the same direction, across the alternating strips, before and after painting. Both the amplitude and time delay of the reflected Gaussian-envelope pulse with a center frequency of $135 \mathrm{GHz}$ were recorded. The results are plotted in Fig. 9(b) and (c).

For easy comparison, a common scale is used on the $x$-axis throughout Fig. 9. The specimen was then painted using a conventional off-the-shelf fast drying acrylic paint, and the thickness of the coating, measured as the difference in the total thickness of the specimen before and after painting, was found to be about $200 \mu \mathrm{m}$. As shown clearly in Fig. 9(b), corrosion reduces the reflectivity of a metal, a fact that can be used for the detection of corroded areas using two-dimensional (2-D) mapping, as is done in [37] and [38]. The rust under the paint can

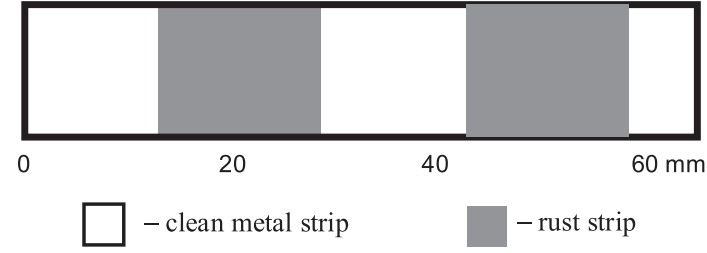

(a)

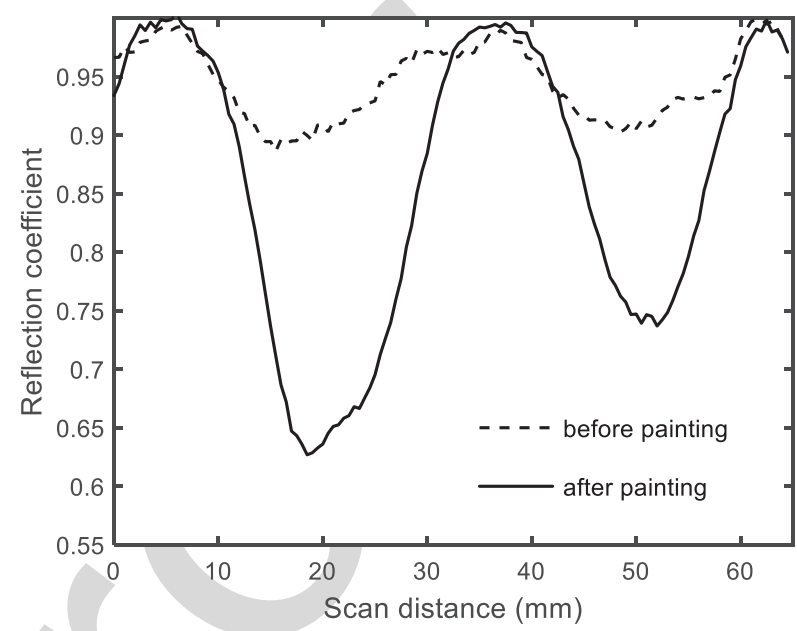

(b)

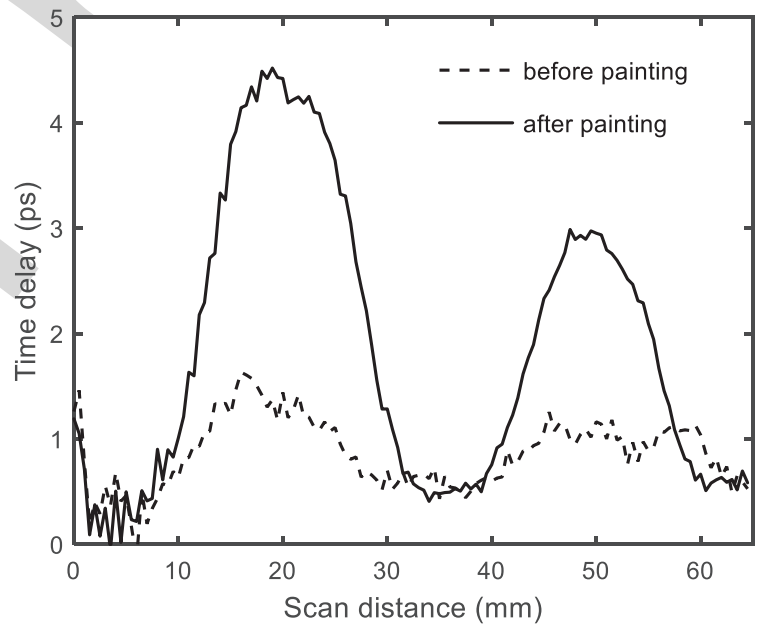

(c)

Fig. 9. Inspection of corrosion under paint by the interferometric enhancement technique. (a) Scheme of the specimen under test with rusty and clean metal areas. (b) Amplitude of the reflected Gaussian-envelope pulse versus scan distance. (c) Time delay of the same pulse versus scan distance.

be detected even more easily due to the stronger scattering of the incident wave on the rough surface caused by the rust. Along with this effect, one can observe that the Gaussian-envelope pulse reflected from the rusted area has been delayed by $3-5$ ps [see Fig. 9(c)]. This delay correlates well with the presence of the rust under the paint and considerably exceeds the time delay that could be expected from a round-trip propagation of the electromagnetic wave in the paint layer. Thus, the additional round-trip time delay in a paint layer with a refraction index $n_{p} \approx 1.6$ [39] and a thickness $d=0.2 \mathrm{~mm}$ can be estimated to be $2 d\left(n_{p}-1\right) \cong 0.8$ ps which is $4-6$ times smaller than the time delay measured in the experiment. 
This anomalous behavior of the time delay can be explained by the interferometric enhancement technique. It can be deduced from the previous analysis that the sweep range of the time of flight of the reflected pulse depends on the ratio of the coefficients of reflection from the dielectric layer interfaces. The closer the amplitudes of the interfering wavelets are, the larger will be the range of the time-delay sweep (naturally within the above-mentioned limits $\pm 1 / 2$ FWHM). In particular, it is for this reason that the sweep of the curve for the alumina slide in Fig. 5 is much larger than that for the quartz slide.

In the case of rust under paint, the decrease in the coefficient of reflection from the paint-metal interface caused by the rust also drives the amplitude of the corresponding wavelet closer to that of the wavelet reflected from the air-paint interface. Accordingly, the time delay of the resultant wavelet in Fig. 9(c) is enhanced by interferometry and this enhancement correlates with the drop in reflection coefficient in Fig. 9(b). Thus simultaneous measurement of both the amplitude and time delay of the sub-THz pulse with Gaussian envelope provides more information about the rusted area and shows still greater promise as a reliable tool for the detection of corrosion under paint.

\section{CONCLUSION}

The technique of interferometry-enhanced TDI presented here is intended for millimeter-wave and THz imaging systems based on Gaussian-envelope wavelet sources. One source of this kind is used here, but the technique can also be implemented with wavelet generators employing other principles such as edge- and pulse-combining methods, RTD generators, and others that are being rapidly developed nowadays. Compact and affordable TDI systems using solid-state sources and detectors promise to move $\mathrm{THz}$ imaging from the research lab to real-world applications, e.g., to clinical intraoperational diagnostics. The technique can also be applied to some nondestructive inspection problems as demonstrated in the example of the detection of corroded areas under paint.

\section{ACKNOWLEDGMENT}

The authors would like to thank Dr. J. Näpänkangas and Dr. R.Blanco-Sequeiros of Oulu University Hospital for providing ex vivo tissue samples for the $\mathrm{THz}$ measurements. Permission for the use of the breast cancer tissue samples (reg. no. 59-2009) was obtained from the Ethical Board of Oulu University Hospital, Finland.

\section{REFERENCES}

[1] D. M. Mittleman, "Twenty years of terahertz imaging [Invited]," Opt. Express, vol. 26, pp. 9417-9431, Apr. 2018, doi: 10.1364/OE.26.009417.

[2] D. Saeedkia, Handbook of Terahertz Technology for Imaging, Sensing and Communications. Cambridge, U.K.: Woodhead, 2013.

[3] D. Suzuki, S. Oda, and Y. Kawano, "A flexible and wearable terahertz scanner," Nature Photon., vol. 10, pp. 809-813, Dec. 2016, doi: 10.1038/nphoton.2016.209.

[4] I. Amenabar, F. Lopez, and A. Mendikute, "In introductory review to THz non-destructive testing of composite mater," J. Infrared Millim. THz Waves, vol. 34, pp. 152-169, Feb. 2013, doi: 10.1007/s10762-012-9949-z.
[5] F. Ellrich et al., "Terahertz quality inspection for automotive and aviation industries," J. Infrared Millim. THz Waves, vol. 41, pp. 470-489, 2020, doi: 10.1007/s10762-019-00639-4.

[6] K. Cooper et al., "THz imaging radar for standoff personnel screening," IEEE Trans. THz Sci. Technol., vol. 1, no. 1, pp. 169-182, Sep. 2011.

[7] W.-H. Lee and W. Lee, "Food inspection system using terahertz imaging," Microw. Opt. Techn. Lett., vol. 56, pp. 1211-1214, May 2014, doi: $10.1002 /$ mop. 28303 .

[8] Y. Sun et al., "A promising diagnostic method: Terahertz pulsed imaging and spectroscopy," World J. Radiol., vol. 3, pp. 55-65, Mar. 2011, doi: $10.4329 / 2$ Fwjr.v3.i3.55.

[9] E. Pickwell-MacPherson and V. P. Wallace, "Terahertz pulsed imaging-A potential medical imaging modality?" Photodiagnosis Photodyn. Therapy, vol. 6, pp. 128-134, Jun. 2009, doi: 10.1016/j.pdpdt.2009.07.002.

[10] Q. Sun et al., "Recent advances in terahertz technology for biomedical applications," Quant. Imag. Med. Surg., vol. 7, pp. 345-355, Jun. 2017, doi: 10.21037/qims.2017.06.02.

[11] U. A. Khan et al., "Broadband dielectric characterization of tumorous and nontumorous breast tissues," IEEE Trans. Microw. Theory Techn., vol. 55, no. 12 , pp. 2887-2893, Dec. 2007.

[12] T. Bowman, M. El-Shenawee, and L. K. Campbell, "Terahertz transmission vs reflection imaging and model-based characterization for excised breast carcinomas," Biomed. Opt. Express, vol. 7, no. 9, pp. 3756-3783, Sep. 2016, doi: 10.1364/BOE.7.003756.

[13] Z. Taylor et al., "THz medical imaging: In vivo hydration sensing," IEEE Trans. THz Sci. Technol., vol. 1, no. 1, pp. 201-219, Sep. 2011.

[14] A. Fitzgerald et al., "Terahertz pulsed imaging of human breast tumors," Radiology, vol. 239, no. 2, pp. 533-540, May 2006, doi: 10.1148/radiol.2392041315.

[15] C.Y. Jen and C. Richter, "Sample thickness measurement with THz-TDS: Resolution and implications," J. Infrared Millim. THz Waves, vol. 35, pp. 840-859, Oct. 2014, doi: 10.1007/s10762-014-0093-9.

[16] K. Su, Y.-C. Shen, and J. A. Zeitler, "Terahertz sensor for non-contact thickness and quality measurement of automobile paints of varying complexity," IEEE Trans. THz Sci. Technol., vol. 4, no. 4, pp. 432-439, Jul. 2014.

[17] S. Krimi et al., "Highly accurate thickness measurement of multi-layered automotive paints using terahertz technology," Appl. Phys. Lett., vol. 109, no. 2, Jul. 2016, Art. no. 021105, doi: 10.1063/1.4955407.

[18] J. van Mechelen, A. Kuzmenko, and H. Merbold, "Stratified dispersive model for material characterization using terahertz time-domain spectroscopy," Opt. Lett., vol. 39, no. 13, pp. 3853-3856, Jul. 2014, doi: 10.1364/OL.39.003853.

[19] Q. Cassar et al., "Iterative tree algorithm to evaluate terahertz signal contribution of specific optical paths within multilayered materials," IEEE Trans. THz Sci. Technol., vol. 9, no. 6, pp. 684-694, Nov. 2019

[20] P. Hillger, J. Grzyb, R. Jain, and U. R. Pfeiffer, "Terahertz imaging and sensing applications with silicon-based technologies," IEEE Trans. THz Sci. Technol., vol. 9, no. 1, pp. 1-19, Jan. 2019.

[21] K. Song, J. Kim, D. Kim, J. Yoo, and J.-S. Rieh, "A 300-GHz CMOS 7-by-7 detector array for optics-less $\mathrm{THz}$ imaging with scan-less target object," J. Infrared Millim. THz Waves, vol. 41, pp. 202-214, Feb. 2020, doi: 10.1007/s10762-019-00664-3.

[22] D. Wang et al., " $240-\mathrm{GHz}$ four-channel power-tuning heterodyne sensing readout system with reflection and transmission measurements in a 130-nm SiGe BiCMOS technology," IEEE Trans. Microw. Theory Techn., vol. 67, no. 12, pp. 5296-5306, Dec. 2019.

[23] M. Bauer et al., "A high-sensitivity AlGaN/GaN HEMT terahertz detector with integrated broadband bow-tie antenna," IEEE Trans. THz Sci. Technol., vol. 9, no. 4, pp. 430-444, Jul. 2019.

[24] A. Arbabian, S. Callender, S. Kang, M. Rangwala, and A. M. Niknejad, "A $94 \mathrm{GHz}$ mm-wave-to-baseband pulsed-radar transceiver with applications in imaging and gesture recognition," IEEE J. Solid-State Circuits, vol. 48, no. 4, pp. 1055-1071, Apr. 2013.

[25] B. P. Ginsburg et al., "A $160 \mathrm{GHz}$ pulsed radar transceiver in $65 \mathrm{~nm}$ CMOS," IEEE J. Solid-State Circuits, vol. 49, no. 4, pp. 984-995, Apr. 2014.

[26] Y. Kawano et al., "RF chipset for impulse UWB radar using $0.13 \mu \mathrm{m}$ InPHEMT technology," IEEE Trans. Microw. Theory Techn., vol. 54, no. 12, pp. 4489-4497, Dec. 2006.

[27] B. B. M. W. Badalawa and M. Fujishima, "60 GHz CMOS pulse generator," Electron. Lett., vol. 43, no. 2, pp. 100-102, Jan. 2007, doi: 10.1049/el:20073324. 
[28] C. Fang, C. L. Law, and J. Hwang, "High-voltage high-efficiency ultrawideband pulse synthesizer," IEEE Microw. Wireless Compon. Lett., vol. 20, no. 1, pp. 49-51, Jan. 2010.

[29] M. M. Assefzadeh and A. Babakhani, "Broadband oscillator-free THz pulse generation and radiation based on direct digital-to-impulse architecture," IEEE J. Solid-State Circuits, vol. 52, no. 11, pp. 2905-2919, Nov. 2017.

[30] M. Egard et al., "In0.53Ga0.47As RTD-MOSFET millimeter-wave wavelet generator," IEEE Electron Device Lett., vol. 33, no. 7, pp. 970-972, Jul. 2012.

[31] I. Vakili, L. Ohlsson, L.-E. Wernersson, and M. Gustafsson, "Time-domain system for millimeter-wave material characterization," IEEE Trans. Microw. Theory Techn., vol. 63, no. 9, pp. 2915-2922, Sep. 2015.

[32] S. N. Vainshtein et al., "Interferometrically enhanced sub-terahertz picosecond imaging utilizing a miniature collapsing-field-domain source," Appl. Phys. Lett., vol. 112, no. 19, May 2018, Art. no. 191104, doi: 10.1063/1.5022453.

[33] S. N. Vainshtein et al., "Collapsing-field-domain-based $200 \mathrm{GHz}$ solidstate source," Appl. Phys. Lett., vol. 115, no. 12, Sep. 2019, Art. no. 123501, doi: $10.1063 / 1.5091616$.

[34] R. M. Azzam and N. M. Bashara, Ellipsometry and Polarized Light. New York, NY, USA: North Holland, 1977.

[35] B. C. Q. Truong, H. D. Tuan, A. J. Fitzgerald, V. P. Wallace, and H. T. Nguyen, "A dielectric model of human breast tissue in terahertz regime," IEEE Trans. Biomed. Eng., vol. 62, no. 2, pp. 699-707, Feb. 2015.

[36] E. A. J. Marcatili and R. A. Schmeltzer, "Hollow metallic and dielectric waveguides for long distance optical transmission and lasers," Bell Syst. Techn. J., vol. 43, pp. 1783-1809, Jul. 1964, doi: 10.1002/j.15387305.1964.tb04108.x.

[37] N. Fuse and T. Fukuchi, "Estimation of surface scattering effect on terahertz non-contact detection of underfilm corrosion," IEEJ Trans. Fundam. Mater., vol. 137, pp. 158-164, Mar. 2017, doi: 10.1541/ieejfms.137.158.

[38] N. Fuse, T. Fukuchi, T. Takahashi, M. Mizuno, and K. Fukunaga, "Evaluation of applicability of noncontact analysis methods to detect rust regions in coated steel plates," IEEE Trans. THz Sci. Technol., vol. 2, no. 2, pp. 242-249, Mar. 2012.

[39] Y.-S. Jin, G.-J. Kim, and S.-G. Jeon, "Terahertz dielectric properties of polymers," J. Korean Phys. Soc., vol. 49, no. 2, pp. 513-517, 2006.

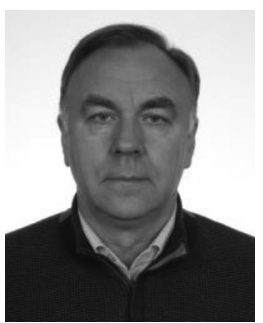

Valeri A. Mikhnev (Senior Member, IEEE) graduated from the Faculty of Radio Physics, Belarusian State University, Minsk, Belarus, in 1979, and the Ph.D. and D.Sc. degrees from the Institute of Applied Physics, National Academy of Sciences of Belarus, Minsk, Belarus, in 1986 and 2003, respectively.

During 1979-2011, he was a Researcher, then the Head of the Laboratory of Microwave Methods for Nondestructive Testing with the Institute of Applied Physics, National Academy of Sciences of Belarus. During 2011-2013, he was a Researcher with Aalto University, Espoo, Finland, where he studied subsurface radar signal processing. During 2014-2019, he was a Visiting Scientist with the University of Oulu, Oulu, Finland, where he was involved in the development of pulsed terahertz imaging systems. His current research interests include millimeter-wave imaging and material characterization.

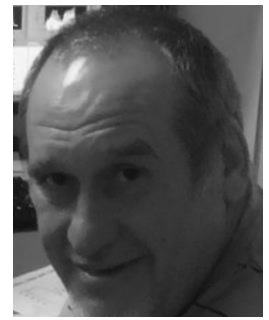

Sergey N. Vainshtein was born in Kostroma, USSR, in 1955. He received the M.Sc. degree (with honors) in physics from Leningrad Polytechnic Institute, Leningrad, USSR, in 1978, and the Ph.D. degree in semiconductor physics from the A. F. Ioffe Institute of Physics and Technology, St. Petersburg, Russia, in 1987.

From 1980 to 2007, he was with the A. F. Ioffe Institute of Physics and Technology. Since 1995, he has been a Senior Scientist with CAS Group, ITEE Department, University of Oulu, Oulu, Finland. His current research interests include pulsed all-electronic $\mathrm{THz}$ emitters, high-speed semiconductor devices based on avalanche switching, picosecond kinetics of laser diodes and light emitting diodes, and original pulsing circuits for their pumping.
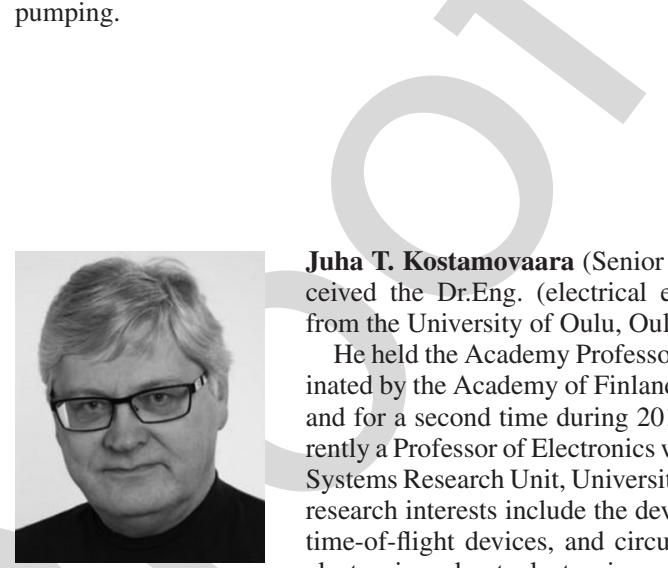

Juha T. Kostamovaara (Senior Member, IEEE) received the Dr.Eng. (electrical engineering) degree from the University of Oulu, Oulu, Finland, in 1987.

He held the Academy Professorship Position nominated by the Academy of Finland during 2006-2011 and for a second time during 2012-2017. He is currently a Professor of Electronics with the Circuits and Systems Research Unit, University of Oulu. His main research interests include the development of pulsed time-of-flight devices, and circuits and systems for electronic and optoelectronic measurements. 